\title{
Robust Bayesian Analysis of Generalized Half Logistic Distribution
}

\author{
Ajit Chaturvedi, Taruna Kumari* \\ Department of Statistics, University of Delhi, India
}

(Received: 5 January 2017; Accepted: 16 May 2017)

\begin{abstract}
In this paper, Robust Bayesian analysis of the generalized half logistic distribution (GHLD) under an $\epsilon$ contamination class of priors for the shape parameter $\lambda$ is considered. ML-II Bayes estimators of the parameters, reliability function and hazard function are derived under the squared-error loss function (SELF) and linear exponential (LINEX) loss function by considering the Type II censoring and the sampling scheme of Bartholomew(1963). Both the cases when scale parameter is known and unknown is considered under Type II censoring and under the sampling scheme of Bartholomew. Simulation study and analysis of a real data set are presented.
\end{abstract}

Keywords GHLD; $\epsilon$-contamination class of prior distributions, ML-II posterior density, Reliability function, Hazard function, SELF, LINEX loss function, Type II censoring, Sampling scheme by Bartholomew, Markov Chain Monte Carlo (MCMC) procedure, Metropolis-Hastings algorithm, Gibbs sampling technique

AMS 2010 subject classifications 62N05, 62F15, 62G35

DOI: $10.19139 /$ soic.v5i2.268

\section{Introduction and preliminaries}

Half logistic has been used in many reliability and survival analysis (especially when the data is censored). Inferences for the half logistic distribution (HLD) have been discussed by several authors. Balakrishnan (1985) discussed on order statistics from the HLD. Balakrishnan and Puthenpura (1986) introduced the best linear unbiased estimators of location and scale parameters of the HLD through linear functions of order statistics. Balakrishnan and Asgharzadeh (2005) gave the inferences for the scaled HLD based on progressively Type II censored samples. Balakrishnan and Hossain (2007) considered generalized (Type II) version of logistic distribution and derived some interesting properties of the distribution. Arora, Bhimani and Patel (2010) obtained the maximum likelihood estimator of the shape parameter in a GHLD based on Type I progressive censoring with varying failure rates. Asgharzadeh, Rezaie and Abdi (2011) compared the methods of estimation for the HLD. Kim, Kang and Seo (2011) proposed the Bayes estimators of the shape parameter and reliability function for the GHLD based on progressively Type II censored data under various loss functions. Seo, Lee and Kang (2012) developed an entropy estimation method for the upper record values from the GHLD. Azimi (2013) derived the Bayes estimators of the shape parameter and the reliability function for the GHLD based on Type II doubly censored samples. Seo and Kang (2014) derived the entropy of GHLD by using the Bayes estimators of an unknown parameter based on Type II censored samples. They also compared these estimators in terms of the mean square error and the bias. Recently, Chaturvedi, Kang and Pathak (2016) developed estimation and testing procedures for the powers of the parameter and the reliability function under Type II censoring and under the sampling scheme of Bartholomew (1963).

*Correspondence to: Taruna Kumari (Email: taruna1986@yahoo.com). Department of Statistics, University of Delhi, Delhi 110007, India.

ISSN 2310-5070 (online) ISSN 2311-004X (print)

Copyright (C) 2017 International Academic Press 
The robust Bayesian viewpoint, since Good (1950), assumed that the subjective information can be quantified in terms of a class $\Gamma$ of possible distributions. The goal is to make inferences or decisions which are robust or flexible over $\Gamma$, i.e., which are relatively insensitive to derivations as the prior distribution varies over $\Gamma$. For a brief review of the literature on robustness in Bayesian model, one may refer to Jeffreys (1961), Box and Tiao (1962), Good (1965, 1967, 1980), Lindley (1961), Dempster (1976), Box (1980).

Bayesian analysis based on $\epsilon$-contamination class of priors has been extensively studied by Berger (1982, 1983), Berger and Berliner (1983), Chaturvedi (1993, 1998), Chaturvedi, Pati and Tomer (2013). This class is a sensible class in the light of prior elicitation process for removing prior judgment error. Betro (2009) provides some numerical procedures for robust Bayesian analysis. For a detailed review on Bayesian robustness one may refer to Berger et al. (2000).

The randome variable $(r v) X$ is said to follow GHLD if its probability density function (pdf) is given by

$$
f(x ; \lambda, \sigma)=\frac{\lambda}{\sigma}\left(\frac{2 e^{-x / \sigma}}{1+e^{-x / \sigma}}\right)^{\lambda} \frac{1}{1+e^{-x / \sigma}}, \quad x>0, \lambda, \sigma>0 .
$$

The reliability function is

$$
\begin{aligned}
R(t) & =P(X>t) \\
& =\left(\frac{1+e^{t / \sigma}}{2}\right)^{-\lambda}
\end{aligned}
$$

and the hazard-rate comes out to be

$$
\begin{aligned}
h(t) & =\frac{f(t ; \lambda)}{R(t)} \\
& =\frac{\lambda}{\sigma\left(1+e^{-t / \sigma}\right)} .
\end{aligned}
$$

Form (1.3), we conclude that the GHLD is fit for the situation when we have increasing failure-rate.

The purpose of the present paper is to give robust Bayesian analysis of parameters, hazard function and reliability function of GHLD. In Section 2 and 4 respectively, for known scale parameter, we obtained the robust Bayes estimators of these parametric functions under SELF and LINEX loss functions considering Type II censoring and the sampling scheme of Bartholomew (1963). In Section 3 and 5 respectively, we consider the case when both shape and scale parameters are unknown. In Section 6, simulation study is performed by using Monte Carlo simulation method, when scale parameter is known. In Section 7, analysis of a real data set using MCMC and Gibbs sampling technique is performed, for the case when both the parameters are unknown. Finally, in Section 8, discussion and some concluding remarks are presented.

\section{Robust Bayesian analysis under Type II censoring with known scale parameter}

Let $n$ items are put on a life test and the test is terminated after $r^{\text {th }}$ failure, so that, $(n-r)$ items survived. Let us denote by $0<X_{(1)} \leq X_{(2)} \leq \ldots \leq X_{(r)}, 0<r<n$, the lifetimes of first $r$ failures. Denoting by $S_{\sigma}=$ $\sum_{i=1}^{r} \ln \left(\frac{1+e^{X_{(i)} / \sigma}}{2}\right)+(n-r) \ln \left(\frac{1+e^{X_{(r)} / \sigma}}{2}\right)$ and $\underline{\mathbf{x}}=\left(x_{(1)}, x_{(2)}, \ldots, x_{(r)}\right)$, the likelihood function is [Seo, Kim and Kang (2013)].

$$
l(\underline{\mathbf{x}} \mid \lambda, \sigma)=\frac{n !}{(n-r) !}\left(\frac{\lambda}{\sigma}\right)^{r}\left(\prod_{i=1}^{r} \frac{1}{1+e^{-x_{(i)} / \sigma}}\right) \exp \left(-\lambda S_{\sigma}\right) .
$$

For the prior density, we consider the following $\epsilon$-contamination class of prior distribution for $\lambda$ [Berger (1985)].

$$
\Gamma=\left\{\pi(\lambda): \pi(\lambda)=(1-\epsilon) g_{o}\left(\lambda \mid \mu_{o}\right)+\epsilon g(\lambda \mid \mu) ; g(\lambda \mid \mu) \in G\right\}
$$


where $\epsilon(0 \leq \epsilon \leq 1)$ is pre-assigned and represents the probability of error in the elicitation of the prior $g_{o}\left(\lambda \mid \mu_{o}\right)$. We consider the base prior, a natural conjugate prior, given by the pdf

$$
g_{o}\left(\lambda \mid \mu_{o}\right)=\frac{\mu_{o}^{\nu}}{\Gamma(\nu)} \lambda^{\nu-1} \exp \left(-\mu_{o} \lambda\right) ; \quad \mu_{o}>0, \nu>0,
$$

where $\left(\mu_{o}, \nu\right)$ represents the vector of hyper parameters. The contamination class $G$ is the class of all natural conjugate priors with the vector of hyper parameters $(\mu, \nu)$, which is given as

$$
G=\left\{g(\lambda \mid \mu)=\frac{\mu^{\nu}}{\Gamma(\nu)} \lambda^{\nu-1} \exp (-\mu \lambda) ; \mu \in\left(\mu_{o}, \infty\right)\right\} .
$$

The predictive density corresponding to the prior $g(\lambda \mid \mu)$ is

$$
m(\underline{\mathbf{x}} \mid g)=\int_{0}^{\infty} l(\lambda \mid \underline{\mathbf{x}}) g(\lambda \mid \mu) d \lambda .
$$

Using (2.1) and (2.4), we obtain from (2.5) that

$$
m(\underline{\mathbf{x}} \mid g)=\frac{n !}{(n-r) ! \sigma^{r}}\left(\prod_{i=1}^{r} \frac{1}{1+e^{-x_{(i)} / \sigma}}\right) \frac{\mu^{\nu}}{\Gamma(\nu)} \frac{\Gamma(r+\nu)}{\left(S_{\sigma}+\mu\right)^{r+\nu}} .
$$

The predictive density corresponding to the base prior is obtained from (2.6) when $\mu=\mu_{o}$. Now, using (2.2), the predictive density corresponding to the generic prior $\pi \in \Gamma$ is

$$
m(\underline{\mathbf{x}} \mid \pi)=(1-\epsilon) m\left(\underline{\mathbf{x}} \mid g_{o}\right)+\epsilon m(\underline{\mathbf{x}} \mid g) .
$$

In the ML-II process, we choose the value of the unknown hyper parameter $\mu$ in a data dependent fashion by maximizing the predictive density $m(\underline{\mathbf{x}} \mid \pi)$ over the class of all prior $\pi \in \Gamma$. Since $g_{o}$ is fixed, we have

$$
\sup _{\pi \in \Gamma} m(\underline{\mathbf{x}} \mid \pi)=(1-\epsilon) m\left(\underline{\mathbf{x}} \mid g_{o}\right)+\epsilon \sup _{g \in G} m(\underline{\mathbf{x}} \mid g)
$$

and $m(\underline{\mathbf{x}} \mid g)$ is maximized when we replace $\mu$ by its maximum likelihood estimator (MLE), which is given by

$$
\hat{\mu}=\max \left\{\mu_{o}, \frac{\nu S_{\sigma}}{r}\right\}
$$

in $g(\lambda \mid \mu)$. Then we have

$$
g(\lambda \mid \hat{\mu})= \begin{cases}\frac{\lambda^{\nu-1}}{\Gamma(\nu)}\left(\frac{\nu S_{\sigma}}{r}\right)^{\nu} \exp \left(\frac{-\nu S_{\sigma}}{r} \lambda\right)=\hat{g}, & \mu_{o}<\frac{\nu S_{\sigma}}{r}, \\ g\left(\lambda \mid \mu_{o}\right)=g_{o}, & \mu_{o} \geq \frac{\nu S_{\sigma}}{r} .\end{cases}
$$

Thus, the ML-II prior density is given by

$$
\hat{\pi}(\lambda)=(1-\epsilon) g_{o}\left(\lambda \mid \mu_{o}\right)+\epsilon \hat{g}(\lambda \mid \hat{\mu}) .
$$

From Berger and Berliner (1983), the ML-II posterior of $\lambda$ is obtained as

$$
\hat{\pi}^{*}(\lambda)=\hat{\eta} g_{o}^{*}(\lambda)+(1-\hat{\eta}) \hat{g}^{*}(\lambda),
$$

where

$$
\begin{aligned}
g_{o}^{*}(\lambda) & =\frac{l(\lambda \mid \underline{\mathbf{x}}) g_{o}\left(\lambda \mid \mu_{o}\right)}{\int_{0}^{\infty} l(\lambda \mid \underline{\mathbf{x}}) g_{o}\left(\lambda \mid \mu_{o}\right) d \lambda} \\
& =\frac{\left(S_{\sigma}+\mu_{o}\right)^{r+\nu}}{\Gamma(r+\nu)} \lambda^{r+\nu-1} \exp \left(-\lambda\left(S_{\sigma}+\mu_{o}\right)\right), \quad \mu_{o} \geq \frac{\nu S_{\sigma}}{r} .
\end{aligned}
$$


Similarly, we get

$$
\hat{g}^{*}(\lambda)= \begin{cases}\frac{\lambda^{r+\nu-1}}{\Gamma(r+\nu)}\left(1+\frac{\nu}{r}\right)^{r+\nu} S_{\sigma}^{r+\nu} \exp \left(-\lambda S_{\sigma}\left(1+\frac{\nu}{r}\right)\right), & \mu_{o}<\frac{\nu S_{\sigma}}{r} \\ g_{o}^{*}(\lambda), & \mu_{o} \geq \frac{\nu S_{\sigma}}{r}\end{cases}
$$

and

$$
\begin{aligned}
\hat{\eta} & =\frac{(1-\epsilon) m\left(\underline{\mathbf{x}} \mid g_{o}\right)}{(1-\epsilon) m\left(\underline{\mathbf{x}} \mid g_{o}\right)+\epsilon m(\underline{\mathrm{x}} \mid \hat{g})} \\
& = \begin{cases}{\left[1+\frac{\epsilon}{(1-\epsilon)} \frac{\nu^{\nu} r^{r}}{S_{\sigma}^{r} \mu_{o}^{\nu}} \frac{\left(S_{\sigma}+\mu_{o}\right)^{r+\nu}}{(r+\nu)^{(r+\nu)}}\right]^{-1},} & \mu_{o}<\frac{\nu S_{\sigma}}{r}, \\
(1-\epsilon), & \mu_{o} \geq \frac{\nu S_{\sigma}}{r} .\end{cases}
\end{aligned}
$$

\subsection{Under SELF}

Now we derive the ML-II estimators of the powers of $\lambda$, hazard-rate and reliability function under SELF. We also derive the expression for posterior variance. It is well known that the Bayes estimator of any parametric function under SELF is its posterior mean. Also the well known form of SELF for estimating $\lambda$ through its estimator $\hat{\lambda}$ is given by

$$
L(\hat{\lambda})=(\hat{\lambda}-\lambda)^{2}
$$

Let us first obtain the Bayes estimator $\lambda^{p}$ ( $\mathrm{p}$ is finite and non zero) and its posterior variance in the following theorem.

Theorem 1

The ML-II posterior mean and variance of $\lambda^{p}$ are given, respectively by

$$
E_{\hat{\pi}^{*}}\left(\lambda^{p}\right)= \begin{cases}\frac{\Gamma(r+\nu+p)}{(r+\nu)}\left\{\hat{\eta}\left(S_{\sigma}+\mu_{o}\right)^{-p}+(1-\hat{\eta})\left(1+\frac{v}{r}\right)^{-p} S_{\sigma}^{-p}\right\}, & \mu_{o}<\frac{\nu S_{\sigma}}{r}, \\ \frac{\Gamma(r+\nu+p)}{(r+\nu)}\left(S_{\sigma}+\mu_{o}\right)^{-p}, & \mu_{o} \geq \frac{\nu S_{\sigma}}{r}\end{cases}
$$

and

$$
V_{\hat{\pi}^{*}}\left(\lambda^{p}\right)=\left\{\begin{array}{rlr}
\left\{\frac{\Gamma(r+\nu+2 p)}{(r+\nu)}-\left(\frac{\Gamma(r+\nu+p)}{(r+\nu)}\right)^{2}\right\} & \\
& \cdot\left\{\hat{\eta}\left(S_{\sigma}+\mu_{o}\right)^{-2 p}+(1-\hat{\eta})\left(1+\frac{v}{r}\right)^{-2 p} S_{\sigma}^{-2 p}\right\} & \\
& +\hat{\eta}(1-\hat{\eta})\left(\frac{\Gamma(r+\nu+p)}{(r+\nu)}\right)^{2}\left\{\left(S_{\sigma}+\mu_{o}\right)^{-p}-\left(1+\frac{v}{r}\right)^{-p} S_{\sigma}^{-p}\right\}^{2}, & \mu_{o}<\frac{\nu S_{\sigma}}{r}, \\
\left\{\frac{\Gamma(r+\nu+2 p)}{(r+\nu)}-\left(\frac{\Gamma(r+\nu+p)}{(r+\nu)}\right)^{2}\right\}\left(S_{\sigma}+\mu_{o}\right)^{-2 p}, & \mu_{o} \geq \frac{\nu S_{\sigma}}{r} .
\end{array}\right.
$$

Proof

Using (2.9), we have

$$
E_{\hat{\pi}^{*}}\left(\lambda^{p}\right)=\hat{\eta} E_{g_{o}^{*}}\left(\lambda^{p}\right)+(1-\hat{\eta}) E_{\hat{g}^{*}}\left(\lambda^{p}\right) .
$$

For $\mu_{o} \geq \frac{\nu S_{\sigma}}{r}$, we obtain

$$
\begin{aligned}
E_{g_{o}^{*}}\left(\lambda^{p}\right) & =\frac{\left(S_{\sigma}+\mu_{o}\right)^{(r+\nu)}}{\Gamma(r+\nu)} \int_{0}^{\infty} \lambda^{r+p+\nu-1} e^{-\lambda\left(S_{\sigma}+\mu_{o}\right)} d \lambda \\
& =\frac{\Gamma(r+\nu+p)}{\Gamma(r+\nu)}\left(S_{\sigma}+\mu_{o}\right)^{-p}
\end{aligned}
$$


Similarly, for $\mu_{o}<\frac{\nu S_{r}}{r}$, it immediately follows that

$$
E_{\hat{g}^{*}}\left(\lambda^{p}\right)=\frac{\Gamma(r+\nu+p)}{\Gamma(r+\nu)}\left(1+\frac{\nu}{r}\right)^{-p} S_{\sigma}^{-p},
$$

thus (2.13) follows on substituting (2.16) and (2.17) in (2.15).

In order to obtain the posterior variance of $\lambda^{p}$, we use the following expression from Berger(1985)

$$
V_{\hat{\pi}^{*}}\left(\lambda^{p}\right)=\hat{\eta} V_{g_{o}^{*}}\left(\lambda^{p}\right)+(1-\hat{\eta}) V_{\hat{g}^{*}}\left(\lambda^{p}\right)+\hat{\eta}(1-\hat{\eta})\left[E_{g_{o}^{*}}\left(\lambda^{p}\right)-E_{\hat{g}^{*}}\left(\lambda^{p}\right)\right]^{2},
$$

where

$$
\begin{aligned}
V_{g_{o}^{*}}\left(\lambda^{p}\right) & =E_{g_{o}^{*}}\left(\lambda^{2 p}\right)-\left[E_{g_{o}^{*}}\left(\lambda^{p}\right)\right]^{2} \\
& =\left[\frac{\Gamma(r+\nu+2 p)}{(r+\nu)}-\left(\frac{\Gamma(r+\nu+p)}{(r+\nu)}\right)^{2}\right]\left(S_{\sigma}+\mu_{o}\right)^{-2 p}
\end{aligned}
$$

and

$$
\begin{aligned}
V_{\hat{g}^{*}}\left(\lambda^{p}\right) & =E_{\hat{g}^{*}}\left(\lambda^{2 p}\right)-\left[E_{\hat{g}^{*}}\left(\lambda^{p}\right)\right]^{2} \\
& =\left[\frac{\Gamma(r+\nu+2 p)}{(r+\nu)}-\left(\frac{\Gamma(r+\nu+p)}{(r+\nu)}\right)^{2}\right] S_{\sigma}^{-2 p}\left(1+\frac{\nu}{r}\right)^{-2 p},
\end{aligned}
$$

now (2.14) follows on using (2.16), (2.17), (2.19) and (2.20) in (2.18).

\section{Corollary 1}

The Bayes estimator of $\lambda$ when $\mathrm{p}=1$ is given by

$$
\hat{\lambda}= \begin{cases}\hat{\lambda}_{o}+(1-\hat{\eta})\left(\hat{\lambda}_{M}-\hat{\lambda}_{o}\right)^{+}, & \mu_{o}<\frac{\nu S_{\sigma}}{r}, \\ \hat{\lambda}_{o}, & \mu_{o} \geq \frac{\nu S_{\sigma}}{r},\end{cases}
$$

where $\hat{\lambda}_{o}=\frac{(r+\nu)}{\left(S_{\sigma}+\nu_{o}\right)}, \hat{\lambda}_{M}=\frac{r}{S_{\sigma}}$ and $\left(a_{M}-a_{o}\right)^{+}= \begin{cases}a_{M}-a_{o}, & a_{M}>a_{o} \\ 0, & \text { otherwise. }\end{cases}$

\section{Corollary 2}

The posterior variance of $\lambda$ when $\mathrm{p}=1$ is given by

$$
V_{\hat{\pi}^{*}}(\lambda)= \begin{cases}(r+\nu)\left\{\hat{\eta}\left(S_{\sigma}+\mu_{o}\right)^{-2}+(1-\hat{\eta})\left(1+\frac{v}{r}\right)^{-2} S_{\sigma}^{-2}\right. & \\ \left.\quad+\hat{\eta}(1-\hat{\eta})(r+\nu)\left[\left(S_{\sigma}+\mu_{o}\right)^{-1}-\left(1+\frac{v}{r}\right)^{-1} S_{\sigma}^{-1}\right]^{2}\right\}, & \mu_{o}<\frac{\nu S_{\sigma}}{r} \\ (r+\nu)\left(S_{\sigma}+\mu_{o}\right)^{-2}, & \mu_{o} \geq \frac{\nu S_{\sigma}}{r} .\end{cases}
$$

Now in the following Theorem, we obtain the ML-II estimator and posterior variance of the hazard rate function, as follows

\section{Theorem 2}

The ML-II estimator of the hazard rate function $h(t)$ is given by

$$
\widehat{h(t)}= \begin{cases}\hat{h}_{o}(t)+(1-\hat{\eta})\left(\hat{h}_{M}(t)-\hat{h}_{o}(t)\right)^{+}, & \mu_{o}<\frac{\nu S_{\sigma}}{r}, \\ \hat{h}_{o}(t), & \mu_{o} \geq \frac{\nu S_{\sigma}}{r},\end{cases}
$$

where $\hat{h}_{o}(t)=\frac{(r+\nu)}{\sigma\left(1+e^{-t / \sigma}\right)\left(S_{\sigma}+\mu_{o}\right)}$ and $\hat{h}_{M}(t)=\frac{r}{\sigma\left(1+e^{-t / \sigma}\right) S_{\sigma}}$. 
Proof

It follows from the fact that

$$
\begin{aligned}
\widehat{h(t)} & =E_{\hat{\pi}^{*}}(h(t)) \\
& =\frac{1}{\sigma\left(1+e^{-t / \sigma}\right)} E_{\hat{\pi}^{*}}(\lambda) \\
& =\frac{1}{\sigma\left(1+e^{-t / \sigma}\right)}\left\{\hat{\eta} E_{g_{o}^{*}}(\lambda)+(1-\hat{\eta}) E_{\hat{g}^{*}}(\lambda)\right\} .
\end{aligned}
$$

Upon using (2.16) and (2.17) with $p=1$ in (2.23), we get (2.22).

\section{Theorem 3}

The posterior variance of the hazard rate function $h(t)$ is given by

$$
V_{\hat{\pi}^{*}}(h(t))= \begin{cases}\frac{(r+\nu)}{\sigma^{2}\left(1+e^{-t / \sigma}\right)^{2}}\left\{\hat{\eta}\left(S_{\sigma}+\mu_{o}\right)^{-2}+(1-\hat{\eta})\left(1+\frac{v}{r}\right)^{-2} S_{\sigma}^{-2}\right. & \\ \left.+\hat{\eta}(1-\hat{\eta})(r+\nu)\left[\left(S_{\sigma}+\mu_{o}\right)^{-1}-\left(1+\frac{v}{r}\right)^{-1} S_{\sigma}^{-1}\right]^{2}\right\}, & \mu_{o}<\frac{\nu S_{\sigma}}{r}, \\ \frac{(r+\nu)}{\sigma^{2}\left(1+e^{-t / \sigma}\right)^{2}}\left(S_{\sigma}+\mu_{o}\right)^{-2}, & \mu_{o} \geq \frac{\nu S_{\sigma}}{r} .\end{cases}
$$

Proof

Using (1.3), we have

$$
\begin{aligned}
V_{\hat{\pi}^{*}}(h(t)) & =V_{\hat{\pi}^{*}}\left(\frac{\lambda}{\sigma\left(1+e^{-t / \sigma}\right)}\right) \\
& =\frac{1}{\sigma^{2}\left(1+e^{-t / \sigma}\right)^{2}} V_{\hat{\pi}^{*}}(\lambda) .
\end{aligned}
$$

Now, applying (2.21) in (2.25), we get (2.24).

In Theorem 4 and Theorem 5, we will obtain the ML-II estimator and the posterior variance of the reliability function $\mathrm{R}(\mathrm{t})$, respectively.

Theorem 4

The ML-II estimator of the reliability function $\mathrm{R}(\mathrm{t})$ is given by

$$
\widehat{R(t)}= \begin{cases}\hat{R}_{o}(t)+(1-\hat{\eta})\left(\hat{R}_{M}(t)-\hat{R}_{o}(t)\right)^{+}, & \mu_{o}<\frac{\nu S_{\sigma}}{r} \\ \hat{R}_{o}(t), & \mu_{o} \geq \frac{\nu S_{\sigma}}{r}\end{cases}
$$

where $\hat{R}_{o}(t)=\left[1+\frac{\ln \left(\left(1+e^{t / \sigma}\right) / 2\right)}{\left(S_{\sigma}+\mu_{o}\right)}\right]^{-(r+\nu)}$ and $\hat{R}_{M}(t)=\left[1+\frac{r \ln \left(\left(1+e^{t / \sigma}\right) / 2\right)}{S_{\sigma}(r+\nu)}\right]^{-(r+\nu)}$.

Proof

Proceeding in a similar manner as in Theorem 2, we get

$$
\begin{aligned}
\widehat{R(t)} & =E_{\hat{\pi}^{*}}(R(t)) \\
& =\hat{\eta} E_{g_{o}^{*}}(R(t))+(1-\hat{\eta}) E_{\hat{g}^{*}}(R(t)) .
\end{aligned}
$$

Let us denote $\hat{R}_{o}(t)=E_{g_{o}^{*}}(R(t))$ and $\hat{R}_{M}(t)=E_{\hat{g}^{*}}(R(t))$, then the above expression can be written as

$$
\widehat{R(t)}=\hat{\eta} \hat{R}_{o}(t)+(1-\hat{\eta}) \hat{R}_{M}(t) .
$$


Upon solving

$$
\begin{aligned}
\hat{R}_{o}(t) & =\frac{\left(S_{\sigma}+\mu_{o}\right)^{(r+\nu)}}{\Gamma(r+\nu)} \int_{0}^{\infty} \lambda^{r+\nu-1} \exp \left[-\lambda\left\{\ln \left(\left(1+e^{t / \sigma}\right) / 2\right)+S_{\sigma}+\mu_{o}\right\}\right] d \lambda \\
& =\left[1+\frac{\ln \left(\left(1+e^{t / \sigma}\right) / 2\right)}{\left(S_{\sigma}+\mu_{o}\right)}\right]^{-(r+\nu)}
\end{aligned}
$$

and similarly,

$$
\hat{R}_{M}(t)=\left[1+\frac{r \ln \left(\left(1+e^{t / \sigma}\right) / 2\right)}{S_{\sigma}(r+\nu)}\right]^{-(r+\nu)},
$$

on using (2.27) and (2.28) in (2.26) yield the required result.

\section{Theorem 5}

The posterior variance of the reliability function $R(t)$ is given by

$$
V_{\hat{\pi}^{*}}(R(t))= \begin{cases}\hat{\eta}\left[\hat{R}_{o}^{2}(t)-\left(\hat{R}_{o}(t)\right)^{2}\right]+(1-\hat{\eta})\left[\hat{R}_{M}^{2}(t)-\left(\hat{R}_{M}(t)\right)^{2}\right] & \\ +\hat{\eta}(1-\hat{\eta})\left[\hat{R}_{o}(t)-\hat{R}_{M}(t)\right]^{2}, & \mu_{o}<\frac{\nu S_{\sigma}}{r}, \\ \hat{R}_{o}^{2}(t)-\left(\hat{R}_{o}(t)\right)^{2}, & \mu_{o} \geq \frac{\nu S_{\sigma}}{r},\end{cases}
$$

where $\hat{R}_{o}^{2}(t)=\left[1+\frac{2 \ln \left(\left(1+e^{t / \sigma}\right) / 2\right)}{\left(S_{\sigma}+\mu_{o}\right)}\right]^{-(r+\nu)}$ and $\hat{R}_{M}^{2}(t)=\left[1+\frac{2 r \ln \left(\left(1+e^{t / \sigma}\right) / 2\right)}{S_{\sigma}(r+\nu)}\right]^{-(r+\nu)}$.

Proof

Let us denote $\hat{R}_{o}^{2}(t)=E_{g_{o}^{*}}\left(R^{2}(t)\right)$ and $\hat{R}_{M}^{2}(t)=E_{\hat{g}^{*}}\left(R^{2}(t)\right)$. By using (2.27) and (2.28) and proceeding in a similar manner as in the second part of Theorem 1, we get (2.29).

\subsection{Under LINEX}

Let us now derive the ML-II estimators of $\lambda$, hazard-rate and reliability function under LINEX loss function. The expression for the LINEX loss function is given by

$$
L(\Delta)=[\exp (a \Delta)-a \Delta-1], \quad a \neq 0,
$$

where $\Delta=\hat{\lambda}-\lambda$. For $a=1$, the function is quite asymmetric with overestimation being more serious than underestimation. When $a<0,(2.30)$ behaves almost exponential when $\Delta<0$ and almost linear when $\Delta>0$. For small values of $|a|$, the function is almost symmetric and close to SELF, viz.,

$$
L(\Delta)=\frac{a^{2} \Delta^{2}}{2}
$$

Under the LINEX loss function (2.30), he ML-II estimator $\lambda$ is given by

$$
\tilde{\lambda}=-\frac{1}{a} \ln E_{\hat{\pi}^{*}}[\exp (-a \lambda)]
$$

Theorem 6

The ML-II estimator of $\lambda$ is given by

$$
\tilde{\lambda}= \begin{cases}-\frac{1}{a}\left\{\ln \tilde{\tau}_{o}+\ln \left[1+(1-\hat{\eta})\left(\frac{\tilde{\tau}_{M}-\tilde{\tau}_{o}}{\tilde{\tau}_{o}}\right)\right]\right\}, & \mu_{o}<\frac{\nu S_{\sigma}}{r}, \\ -\frac{1}{a} \ln \tilde{\tau}_{o}, & \mu_{o} \geq \frac{\nu S_{\sigma}}{r},\end{cases}
$$

where $\tilde{\tau}_{o}=\left[1+\frac{a}{\left(S_{\sigma}+\mu_{o}\right)}\right]^{-(r+\nu)}$ and $\tilde{\tau}_{M}=\left[1+\frac{a r}{S_{\sigma}(\nu+r)}\right]^{-(r+\nu)}$. 
Proof

Using (2.31),

$$
\tilde{\lambda}=-\frac{1}{a} \ln \left[\hat{\eta} E_{g_{o}^{*}}[\exp (-a \lambda)]+(1-\hat{\eta}) E_{\hat{g}^{*}}[\exp (-a \lambda)]\right] .
$$

Let us denote $\tilde{\tau}_{o}=E_{g_{o}^{*}}[\exp (-a \lambda)]$ and $\tilde{\tau}_{M}=E_{\hat{g}^{*}}[\exp (-a \lambda)]$, then the above expression can be rewritten as

$$
\tilde{\lambda}=-\frac{1}{a} \ln \left[\hat{\eta} \tilde{\tau}_{o}+(1-\hat{\eta}) \tilde{\tau}_{M}\right]
$$

where

$$
\begin{aligned}
\tilde{\tau}_{o} & =\frac{\left(S_{\sigma}+\mu_{o}\right)^{(r+\nu)}}{\Gamma(r+\nu)} \int_{0}^{\infty} \lambda^{r+\nu-1} \exp \left[-\lambda\left(S_{\sigma}+\mu_{o}+a\right)\right] d \lambda \\
& =\left[1+\frac{a}{\left(S_{\sigma}+\mu_{o}\right)}\right]^{-(r+\nu)} .
\end{aligned}
$$

Similarly,

$$
\tilde{\tau}_{M}=\left\{1+\frac{a r}{S_{\sigma}(\nu+r)}\right\}^{-(r+\nu)} .
$$

Upon rewriting (2.34) with (2.35) and (2.36) yield (2.32).

The expectation of the LINEX loss function for $\tilde{\lambda}$ with respect to ML-II posterior distribution of $\lambda$ is

$$
\begin{aligned}
a E_{\hat{\pi}^{*}}(\lambda-\tilde{\lambda}) & =a\left[E_{\hat{\pi}^{*}}(\lambda)-\tilde{\lambda}\right] \\
& = \begin{cases}a\left[\hat{\lambda}_{o}+(1-\hat{\eta})\left(\hat{\lambda}_{M}-\hat{\lambda}_{o}\right)^{+}-\tilde{\lambda}\right], & \mu_{o}<\frac{\nu S_{\sigma}}{r}, \\
a\left[\hat{\lambda}_{o}-\tilde{\lambda}\right], & \mu_{o} \geq \frac{\nu S_{\sigma}}{r} .\end{cases}
\end{aligned}
$$

Next we obtain the ML-II estimator of hazard rate function $\mathrm{h}(\mathrm{t})$ respectively. Proceeding in a similar manner as in Theorem 6 and denoting $\tilde{\phi}_{o}(t)=E_{g_{o}^{*}}[\exp (-a h(t))]$ and $\tilde{\phi}_{M}(t)=E_{\hat{g}^{*}}[\exp (-a h(t))]$, the expressions for ML-II estimator of $h(t)$ under LINEX can be derived as follows.

Theorem 7

The ML-II estimator of the hazard rate function $h(t)$ is given by

$$
\widetilde{h(t)}= \begin{cases}-\frac{1}{a}\left\{\ln \tilde{\phi}_{o}(t)+\ln \left[1+(1-\hat{\eta})\left(\frac{\tilde{\phi}_{M}(t)-\tilde{\phi}_{o}(t)}{\tilde{\phi}_{o}(t)}\right)\right]\right\}, & \mu_{o}<\frac{\nu S_{\sigma}}{r} \\ -\frac{1}{a} \ln \phi_{o}(t), & \mu_{o} \geq \frac{\nu S_{\sigma}}{r}\end{cases}
$$

where $\tilde{\phi}_{o}(t)=\left[1+\frac{a}{\sigma\left(1+e^{-t / \sigma}\right)\left(S_{\sigma}+\mu_{o}\right)}\right]^{-(r+\nu)}$ and $\tilde{\phi}_{M}(t)=\left[1+\frac{a r}{\sigma S_{\sigma}(\nu+r)\left(1+e^{-t / \sigma}\right)}\right]^{-(r+\nu)}$.

The expectation of the LINEX loss function for $\widetilde{h(t)}$ is obtained as

$$
\begin{aligned}
a E_{\hat{\pi}^{*}}(h(t)-\tilde{h}(t)) & =a\left[E_{\hat{\pi}^{*}}(h(t))-\widetilde{h(t)}\right] \\
& = \begin{cases}a\left[\hat{h}_{o}(t)+(1-\hat{\eta})\left(\hat{h}_{M}(t)-\hat{h}_{o}(t)\right)^{+}-\widetilde{h(t)}\right], & \mu_{o}<\frac{\nu S_{\sigma}}{r}, \\
a\left[\hat{h}_{o}(t)-\widetilde{h(t)}\right], & \mu_{o} \geq \frac{\nu S_{\sigma}}{r} .\end{cases}
\end{aligned}
$$

In order to derive the ML-II estimator of the reliability function $R(t)$, let us denote $\tilde{\psi}_{o}(t)=E_{g_{o}^{*}}[\exp (-a R(t))]$ and $\tilde{\psi}_{M}(t)=E_{\hat{g}^{*}}[\exp (-a R(t))]$, then the expressions for ML-II estimator of $R(t)$ under LINEX can be derived as follows. 


\section{Theorem 8}

The ML-II estimator of the reliability function $R(t)$ under LINEX is given by

$$
\widetilde{R(t)}= \begin{cases}-\frac{1}{a}\left\{\ln \tilde{\psi}_{o}(t)+\ln \left[1+(1-\hat{\eta})\left(\frac{\tilde{\psi}_{M}(t)-\tilde{\psi}_{o}(t)}{\tilde{\psi}_{o}(t)}\right)\right]\right\}, & \mu_{o}<\frac{\nu S_{\sigma}}{r}, \\ -\frac{1}{a} \ln \tilde{\psi}_{o}(t), & \mu_{o} \geq \frac{\nu S_{\sigma}}{r}\end{cases}
$$

where

$$
\tilde{\psi}_{o}(t)=\sum_{k=0}^{\infty} \frac{(-a)^{k}}{k !}\left[1+\frac{k \ln \left(\left(1+e^{t / \sigma}\right) / 2\right)}{\left(S_{\sigma}+\mu_{o}\right)}\right]^{-(r+\nu)} \text { and } \tilde{\psi}_{M}(t)=\sum_{k=0}^{\infty} \frac{(-a)^{k}}{k !}\left[1+\frac{k r \ln \left(\left(1+e^{t / \sigma}\right) / 2\right)}{S_{\sigma}(r+\nu)}\right]^{-(r+\nu)} \text {. }
$$

Further, the posterior expectation for the LINEX loss function of $\widetilde{R(t)}$ is given by

$$
\begin{aligned}
a E_{\hat{\pi}^{*}}(R(t)-\widetilde{R(t)}) & =a\left[E_{\hat{\pi}^{*}}(R(t))-\widetilde{R(t)}\right] \\
& = \begin{cases}a\left[\hat{R}_{o}(t)+(1-\hat{\eta})\left(\hat{R}_{M}(t)-\hat{R}_{o}(t)\right)^{+}-\widetilde{R(t)}\right], & \mu_{o}<\frac{\nu S_{\sigma}}{r}, \\
a\left[\hat{R}_{o}(t)-\widetilde{R(t)}\right], & \mu_{o} \geq \frac{\nu S_{\sigma}}{r} .\end{cases}
\end{aligned}
$$

\section{Robust Bayesian analysis under Type II censoring with unknown shape and scale parameters}

In this section we assume that both the parameters of the generalized half logistic distribution are unknown. We assume the following prior distribution to carry out the robust Bayesian analysis for the generalized half logistic distribution by assuming independent priors for both the parameters.

$$
\Gamma=\left\{\pi(\sigma, \lambda): \pi(\sigma, \lambda)=(1-\epsilon) q_{o}+\epsilon q\right\},
$$

where $q_{o}=g_{o}\left(\lambda \mid \mu_{o}\right) P(\sigma)$ and $q=g(\lambda \mid \mu) P(\sigma)$ with $g_{o}$ and $g$ as defined in (2.3) and (2.4), respectively. The density of $P(\sigma)$, the prior for the scale parameter $\sigma$, is assumed to be inverted gamma

$$
P(\sigma)=\frac{\beta^{\alpha}}{\Gamma(\alpha) \sigma^{\alpha+1}} \exp (-\beta / \sigma), \quad \beta>0, \alpha>0 .
$$

The predictive density corresponding to the prior $\pi(\sigma, \lambda)$ comes out to be

$$
m(\underline{\mathbf{x}} \mid \pi)=(1-\epsilon) m\left(\underline{\mathbf{x}} \mid q_{o}\right)+\epsilon m(\underline{\mathbf{x}} \mid q), \quad 0<\lambda<\infty,
$$

where

$$
\begin{aligned}
m(\underline{\mathbf{x}} \mid q) & =\int_{\sigma=0}^{\infty} \int_{\lambda=0}^{\infty} l(\lambda, \sigma \mid \underline{\mathbf{x}}) g(\lambda \mid \mu) P(\sigma) d \lambda d \sigma \\
& =\frac{n !}{(n-r) !} \frac{\Gamma(r+\nu)}{\Gamma(\nu)} \frac{\mu^{\nu} \beta^{\alpha}}{\Gamma(\alpha)} \int_{0}^{\infty} \frac{\sigma^{-\alpha-r-1} \exp (-\beta / \sigma)}{\left(S_{\sigma}+\mu\right)^{r+\nu}}\left(\prod_{i=1}^{r} \frac{1}{1+e^{-x_{(i)} / \sigma}}\right) d \sigma .
\end{aligned}
$$

In order to obtain, the ML-II Bayes estimate of $\lambda$ and $\sigma$ under Type II censoring, we need their marginal posterior under the base prior $q_{o}$ and the generic prior $q$. But, in the present situation it is not possible to get explicit expressions for them. We, therefore, apply Gibbs sampler to get the samples from the marginal distributions of these parameters. For this the full conditionals of $\lambda$ given $\sigma$ is given by

$$
\hat{\pi}^{*}(\lambda \mid \sigma)=\hat{\eta} g_{o}^{*}(\lambda \mid \sigma)+(1-\hat{\eta}) \hat{g}^{*}(\lambda \mid \sigma), \quad 0<\lambda<\infty .
$$

Similarly, by combining the likelihood (2.1) with the prior (3.2), we get the conditional posterior density of $z^{*}(\sigma \mid \lambda)$ as

$$
z^{*}(\sigma \mid \lambda) \propto \sigma^{-\alpha-r-1} \exp \left(-\lambda S_{\sigma}-\beta / \sigma\right)\left(\prod_{i=1}^{r} \frac{1}{1+e^{-x_{(i)} / \sigma}}\right) .
$$




\section{Robust Bayesian analysis under the sampling scheme of Bartholomew with known scale parameter}

Let $X_{(1)} \leq X_{(2)} \leq \ldots \leq X_{(n)}$ be the failure times of $n$ items under test from (1.3). Then test begins at time $X_{(0)}=0$ and the system operates till $X_{(1)}=x_{1}$ when the first failure occurs. The failed item is replaced by a new one and the system operates till the second failure occurs at time $X_{(2)}=x_{2}$ and so on. The experiment is terminated at time $t_{o}$. Here, $X_{(i)}$ is the time until $i^{\text {th }}$ failure measured from time 0 .

\section{Lemma 1}

If $N\left(t_{o}\right)$ be the number of failures during the interval $\left[0, t_{o}\right]$, then

$$
P\left[N\left(t_{o}\right)=r \mid t_{o}\right]=\frac{\left[n \lambda \ln \left(\left(1+e^{t_{o} / \sigma}\right) / 2\right)\right]^{r}}{r !} \exp \left[-n \lambda \ln \left(\left(1+e^{t_{o} / \sigma}\right) / 2\right)\right],
$$

Let us consider for the prior density, the same $\epsilon$-contamination class of prior distribution as in Section 2 . Then the predictive density corresponding to the prior $g(\lambda \mid \mu)$ in the sampling scheme of Bartholomew is given by

$$
m(\underline{\mathbf{x}} \mid g)=\int_{0}^{\infty} P\left[N\left(t_{o}\right)=r \mid t_{o}\right] g(\lambda \mid \mu) d \lambda .
$$

Using (4.1) and (2.4), we obtain from (4.2) that

$$
m(\underline{\mathrm{x}} \mid g)=\left(\begin{array}{c}
r+\nu-1 \\
r
\end{array}\right)\left[\frac{\mu}{n \ln \left(\left(1+e^{t_{o} / \sigma}\right) / 2\right)}\right]^{\nu}\left[1+\frac{\mu}{n \ln \left(\left(1+e^{t_{o} / \sigma}\right) / 2\right)}\right]^{-(r+\nu)} .
$$

The predictive density corresponding to the base prior is obtained from (4.3) when $\mu=\mu_{o}$. Now, as earlier, in the sampling scheme of Bartholomew the predictive density corresponding to the generic prior $\pi \in \Gamma$ is given by (2.7).

As we have already discussed here also we choose the value of the unknown hyper parameter $\mu$ in a data dependent fashion by maximizing the predictive density $m(\underline{\mathrm{x}} \mid \pi)$ over the class of all prior $\pi \in \Gamma$. Since $g_{o}$ is fixed, we have

$$
\sup _{\pi \in \Gamma} m(\underline{\mathbf{x}} \mid \pi)=(1-\epsilon) m\left(\underline{\mathbf{x}} \mid g_{o}\right)+\epsilon \sup _{g \in G} m(\underline{\mathbf{x}} \mid g)
$$

and $m(\underline{\mathbf{x}} \mid g)$ is maximized when we replace $\mu$ by its maximum likelihood estimator (MLE), which is given by

$$
\hat{\mu}=\max \left\{\mu_{o}, \frac{\nu n \ln \left(\left(1+e^{t_{o} / \sigma}\right) / 2\right)}{r}\right\}
$$

in $g(\lambda \mid \mu)$. Then we have

$$
g(\lambda \mid \hat{\mu})= \begin{cases}\frac{\lambda^{\nu-1}}{\Gamma(\nu)}\left(\frac{\nu n \ln \left(\left(1+e^{t_{o} / \sigma}\right) / 2\right)}{r}\right)^{\nu} \exp \left(\frac{-\nu n \ln \left(\left(1+e^{t_{o} / \sigma}\right) / 2\right)}{r} \lambda\right)=\hat{g}, & \mu_{o}<\frac{\nu n \ln \left(\left(1+e^{t_{o} / \sigma}\right) / 2\right)}{r}, \\ g\left(\lambda \mid \mu_{o}\right)=g_{o}, & \mu_{o} \geq \frac{\nu n \ln \left(\left(1+e^{t_{o} / \sigma}\right) / 2\right)}{r} .\end{cases}
$$

Thus, the ML-II prior density is given by

$$
\hat{\pi}(\lambda)=(1-\epsilon) g_{o}\left(\lambda \mid \mu_{o}\right)+\epsilon \hat{g}(\lambda \mid \hat{\mu}) .
$$

From Berger and Berliner (1983), the ML-II posterior of $\lambda$ is obtained as

$$
\hat{\pi}^{*}(\lambda)=\hat{\eta} g_{o}^{*}(\lambda)+(1-\hat{\eta}) \hat{g}^{*}(\lambda),
$$


where for $\mu_{o} \geq \frac{\nu n \ln \left(\left(1+e^{t_{o} / \sigma}\right) / 2\right)}{r}$

$$
\begin{aligned}
g_{o}^{*}(\lambda) & =\frac{P\left[N\left(t_{o}\right)=r \mid t_{o}\right] g_{o}\left(\lambda \mid \mu_{o}\right)}{\int_{0}^{\infty} P\left[N\left(t_{o}\right)=r \mid t_{o}\right] g_{o}\left(\lambda \mid \mu_{o}\right) d \lambda} \\
& =\frac{\left[\mu_{o}+n \ln \left(\left(1+e^{t_{o} / \sigma}\right) / 2\right)\right]^{r+\nu}}{\Gamma(r+\nu)} \lambda^{r+\nu-1} \exp \left[-\lambda\left(\mu_{o}+n \ln \left(\left(1+e^{t_{o} / \sigma}\right) / 2\right)\right)\right] .
\end{aligned}
$$

Similarly, we get

$$
\hat{g}^{*}(\lambda)= \begin{cases}\frac{\lambda^{r+\nu-1}}{\Gamma(r+\nu)}\left(1+\frac{\nu}{r}\right)^{r+\nu}\left[n \ln \left(\left(1+e^{t_{o} / \sigma}\right) / 2\right)\right]^{r+\nu} & \\ \cdot \exp \left[-\lambda n \ln \left(\left(1+e^{t_{o} / \sigma}\right) / 2\right)\left(1+\frac{\nu}{r}\right)\right], & \mu_{o}<\frac{\nu n \ln \left(\left(1+e^{t_{o} / \sigma}\right) / 2\right)}{r} \\ g_{o}^{*}(\lambda), & \mu_{o} \geq \frac{\nu n \ln \left(\left(1+e^{t_{o} / \sigma}\right) / 2\right)}{r}\end{cases}
$$

and

$$
\begin{aligned}
\hat{\eta} & =\frac{(1-\epsilon) m\left(\underline{\mathbf{x}} \mid g_{o}\right)}{(1-\epsilon) m\left(\underline{\mathbf{x}} \mid g_{o}\right)+\epsilon m(\underline{\mathbf{x}} \mid \hat{g})} \\
& = \begin{cases}{\left[1+\frac{\epsilon}{(1-\epsilon)} \frac{\nu^{\nu} r^{r}}{\left[n \ln \left(\left(1+e^{t_{o} / \sigma}\right) / 2\right)\right]^{r} \mu_{o}^{\nu}} \frac{\left[n \ln \left(\left(1+e^{t_{o} / \sigma}\right) / 2\right)+\mu_{o}\right]^{r+\nu}}{(r+\nu)^{(r+\nu)}}\right]^{-1},} & \mu_{o}<\frac{\nu n \ln \left(\left(1+e^{t_{o} / \sigma}\right) / 2\right)}{r}, \\
(1-\epsilon), & \mu_{o} \geq \frac{\nu n \ln \left(\left(1+e^{t_{o} / \sigma}\right) / 2\right)}{r} .\end{cases}
\end{aligned}
$$

\subsection{Under SELF}

Now we state without proofs some results concerning point estimators under the sampling scheme of Bartholomew. The proofs are on similar lines as in Type II censoring case.

Theorem 9

The ML-II posterior mean and variance of $\lambda^{p}$ under SELF are given, respectively by

$$
E_{\hat{\pi}^{*}}\left(\lambda^{p}\right)=\left\{\begin{array}{cl}
\frac{\Gamma(r+\nu+p)}{(r+\nu)}\left\{\hat{\eta}\left[n \ln \left(\left(1+e^{t_{o} / \sigma}\right) / 2\right)+\mu_{o}\right]^{-p}+(1-\hat{\eta})\right. & \\
\left.\cdot\left(1+\frac{v}{r}\right)^{-p}\left[n \ln \left(\left(1+e^{t_{o} / \sigma}\right) / 2\right)\right]^{-p}\right\}, & \mu_{o}<\frac{\nu n \ln \left(\left(1+e^{t_{o} / \sigma}\right) / 2\right)}{r}, \\
\frac{\Gamma(r+\nu+p)}{(r+\nu)}\left[n \ln \left(\left(1+e^{t_{o} / \sigma}\right) / 2\right)+\mu_{o}\right]^{-p}, & \mu_{o} \geq \frac{\nu n \ln \left(\left(1+e^{t_{o} / \sigma}\right) / 2\right)}{r}
\end{array}\right.
$$

and

$$
V_{\hat{\pi}^{*}}\left(\lambda^{p}\right)=\left\{\begin{array}{rlrl}
\left\{\frac{\Gamma(r+\nu+2 p)}{(r+\nu)}-\left(\frac{\Gamma(r+\nu+p)}{(r+\nu)}\right)^{2}\right\}\left\{\hat{\eta}\left[n \ln \left(\left(1+e^{t_{o} / \sigma}\right) / 2\right)+\mu_{o}\right]^{-2 p}\right. & \\
& \left.+(1-\hat{\eta}) \cdot\left(1+\frac{v}{r}\right)^{-2 p}\left[n \ln \left(\left(1+e^{t_{o} / \sigma}\right) / 2\right)\right]^{-2 p}\right\} & \\
& +\hat{\eta}(1-\hat{\eta})\left(\frac{\Gamma(r+\nu+p)}{(r+\nu)}\right)^{2}\left\{\left[n \ln \left(\left(1+e^{t_{o} / \sigma}\right) / 2\right)+\mu_{o}\right]^{-p}\right. & \\
& \left.-\left(1+\frac{v}{r}\right)^{-p}\left[n \ln \left(\left(1+e^{t_{o} / \sigma}\right) / 2\right)\right]^{-p}\right\}^{2}, & \mu_{o}<\frac{\nu n \ln \left(\left(1+e^{t_{o}}\right) / 2\right)}{r}, \\
\left\{\frac{\Gamma(r+\nu+2 p)}{(r+\nu)}-\left(\frac{\Gamma(r+\nu+p)}{(r+\nu)}\right)^{2}\right\}\left[n \ln \left(\left(1+e^{t_{o} / \sigma}\right) / 2\right)+\mu_{o}\right]^{-2 p}, & \mu_{o} \geq \frac{\nu n \ln \left(\left(1+e^{t_{o} / \sigma}\right) / 2\right)}{r} .
\end{array}\right.
$$

Theorem 10

The ML-II estimator of the hazard rate function $\mathrm{h}(\mathrm{t})$ under SELF is given by

$$
\widehat{h(t)}= \begin{cases}\hat{h}_{o}(t)+(1-\hat{\eta})\left(\hat{h}_{M}(t)-\hat{h}_{o}(t)\right)^{+}, & \mu_{o}<\frac{\nu n \ln \left(\left(1+e^{t_{o} / \sigma}\right) / 2\right)}{r}, \\ \hat{h}_{o}(t), & \mu_{o} \geq \frac{\nu n \ln \left(\left(1+e^{t_{o} / \sigma}\right) / 2\right)}{r},\end{cases}
$$


where $\hat{h}_{o}(t)=\frac{(r+\nu)}{\sigma\left(1+e^{-t / \sigma}\right)\left[n \ln \left(\left(1+e^{t_{o} / \sigma}\right) / 2\right)+\mu_{o}\right]}$ and $\hat{h}_{M}(t)=\frac{r}{\sigma\left(1+e^{-t / \sigma}\right)\left[n \ln \left(\left(1+e^{t_{o} / \sigma}\right) / 2\right)\right]}$.

Theorem 11

The posterior variance of the hazard rate function $h(t)$ under SELF is given by

$$
V_{\hat{\pi}^{*}}(h(t))=\left\{\begin{array}{lll}
\frac{(r+\nu)}{\sigma^{2}\left(1+e^{-t / \sigma}\right)^{2}}\left\{\hat{\eta}\left[n \ln \left(\left(1+e^{t_{o} / \sigma}\right) / 2\right)+\mu_{o}\right]^{-2}+(1-\hat{\eta})\right. & \\
\cdot\left(1+\frac{v}{r}\right)^{-2}\left[n \ln \left(\left(1+e^{t_{o} / \sigma}\right) / 2\right)\right]^{-2}+\hat{\eta}(1-\hat{\eta})(r+\nu) & \\
\cdot\left[\left[n \ln \left(\left(1+e^{t_{o} / \sigma}\right) / 2\right)+\mu_{o}\right]^{-1}\right. & \\
\left.\left.-\left(1+\frac{v}{r}\right)^{-1}\left[n \ln \left(\left(1+e^{t_{o} / \sigma}\right) / 2\right)\right]^{-1}\right]^{2}\right\}, & \mu_{o}<\frac{\nu n \ln \left(\left(1+e^{t_{o} / \sigma}\right) / 2\right)}{r}, \\
\frac{(r+\nu)}{\left(1+e^{-t / \sigma}\right)^{2}}\left[n \ln \left(\left(1+e^{t_{o} / \sigma}\right) / 2\right)+\mu_{o}\right]^{-2}, & \mu_{o} \geq \frac{\nu n \ln \left(\left(1+e^{t_{o} / \sigma}\right) / 2\right)}{r} .
\end{array}\right.
$$

Theorem 12

The ML-II estimator of the reliability function $R(t)$ under SELF is given by

$$
\widehat{R(t)}= \begin{cases}\hat{R}_{o}(t)+(1-\hat{\eta})\left(\hat{R}_{M}(t)-\hat{R}_{o}(t)\right)^{+}, & \mu_{o}<\frac{\nu n \ln \left(\left(1+e^{t_{o} / \sigma}\right) / 2\right)}{r}, \\ \hat{R}_{o}(t), & \mu_{o} \geq \frac{\nu n \ln \left(\left(1+e^{t_{o} / \sigma}\right) / 2\right)}{r},\end{cases}
$$

where $\hat{R}_{o}(t)=\left[1+\frac{\ln \left(\left(1+e^{t / \sigma}\right) / 2\right)}{\left[n \ln \left(\left(1+e^{t_{o} / \sigma}\right) / 2\right)+\mu_{o}\right]}\right]^{-(r+\nu)}$ and $\hat{R}_{M}(t)=\left[1+\frac{r \ln \left(\left(1+e^{t / \sigma}\right) / 2\right)}{\left[n \ln \left(\left(1+e^{t_{o}}\right) / 2\right)\right](r+\nu)}\right]^{-(r+\nu)}$.

Theorem 13

The posterior variance of the reliability function $R(t)$ under SELF is given by

$$
V_{\hat{\pi}^{*}}(R(t))= \begin{cases}\hat{\eta}\left[\hat{R}_{o}^{2}(t)-\left(\hat{R}_{o}(t)\right)^{2}\right]+(1-\hat{\eta})\left[\hat{R}_{M}^{2}(t)-\left(\hat{R}_{M}(t)\right)^{2}\right] & \\ +\hat{\eta}(1-\hat{\eta})\left[\hat{R}_{o}(t)-\hat{R}_{M}(t)\right]^{2}, & \mu_{o}<\frac{\nu n \ln \left(\left(1+e^{t_{o} / \sigma}\right) / 2\right)}{r}, \\ \hat{R}_{o}^{2}(t)-\left(\hat{R}_{o}(t)\right)^{2}, & \mu_{o} \geq \frac{\nu n \ln \left(\left(1+e^{t_{o} / \sigma}\right) / 2\right)}{r},\end{cases}
$$

where $\hat{R}_{o}^{2}(t)=\left[1+\frac{2 \ln \left(\left(1+e^{t / \sigma}\right) / 2\right)}{\left[n \ln \left(\left(1+e^{t_{o} / \sigma}\right) / 2\right)+\mu_{o}\right]}\right]^{-(r+\nu)}$ and $\hat{R}_{M}^{2}(t)=\left[1+\frac{2 r \ln \left(\left(1+e^{t / \sigma}\right) / 2\right)}{\left[n \ln \left(\left(1+e^{t_{o} / \sigma}\right) / 2\right)\right](r+\nu)}\right]^{-(r+\nu)}$.

\subsection{Under LINEX}

Theorem 14

The ML-II estimator of $\lambda$ under LINEX is given by

$$
\tilde{\lambda}= \begin{cases}-\frac{1}{a}\left\{\ln \tilde{\tau}_{o}+\ln \left[1+(1-\hat{\eta})\left(\frac{\tilde{\tau}_{M}-\tilde{\tau}_{o}}{\tilde{\tau}_{o}}\right)\right]\right\}, & \mu_{o}<\frac{\nu n \ln \left(\left(1+e^{t_{o} / \sigma}\right) / 2\right)}{r}, \\ -\frac{1}{a} \ln \tilde{\tau}_{o}, & \mu_{o} \geq \frac{\nu n \ln \left(\left(1+e^{t_{o} / \sigma}\right) / 2\right)}{r},\end{cases}
$$

where $\left.\tilde{\tau}_{o}=\left[1+\frac{a}{\left[n \ln \left(\left(1+e^{t_{o} / \sigma}\right) / 2\right)+\mu_{o}\right.}\right]\right]^{-(r+\nu)}$ and $\tilde{\tau}_{M}=\left[1+\frac{a r}{\left[n \ln \left(\left(1+e^{t_{o} / \sigma}\right) / 2\right)\right](\nu+r)}\right]^{-(r+\nu)}$.

Theorem 15

The ML-II estimator of the hazard rate function $h(t)$ under LINEX is given by

$$
\widetilde{h(t)}= \begin{cases}-\frac{1}{a}\left\{\ln \tilde{\phi}_{o}(t)+\ln \left[1+(1-\hat{\eta})\left(\frac{\tilde{\phi}_{M}(t)-\tilde{\phi}_{o}(t)}{\tilde{\phi}_{o}(t)}\right)\right]\right\}, & \mu_{o}<\frac{\nu n \ln \left(\left(1+e^{t_{o} / \sigma}\right) / 2\right)}{r}, \\ -\frac{1}{a} \ln \tilde{\phi}_{o}(t), & \mu_{o} \geq \frac{\nu n \ln \left(\left(1+e^{t_{o} / \sigma}\right) / 2\right)}{r},\end{cases}
$$


where

$\tilde{\phi}_{o}(t)=\left[1+\frac{a}{\sigma\left(1+e^{-t / \sigma}\right)\left[n \ln \left(\left(1+e^{t_{o}}\right) / 2\right)+\mu_{o}\right]}\right]^{-(r+\nu)}$ and $\tilde{\phi}_{M}(t)=\left[1+\frac{a r}{\sigma\left[n \ln \left(\left(1+e^{t_{o} / \sigma}\right) / 2\right)\right](\nu+r)\left(1+e^{-t / \sigma}\right)}\right]^{-(r+\nu)}$.

Theorem 16

The ML-II estimator of the reliability function $R(t)$ under LINEX is given by

$$
\widetilde{R(t)}= \begin{cases}-\frac{1}{a}\left\{\ln \tilde{\psi}_{o}(t)+\ln \left[1+(1-\hat{\eta})\left(\frac{\tilde{\psi}_{M}(t)-\tilde{\psi}_{o}(t)}{\tilde{\psi}_{o}(t)}\right)\right]\right\}, & \mu_{o}<\frac{\nu n \ln \left(\left(1+e^{t_{o} / \sigma}\right) / 2\right)}{r}, \\ -\frac{1}{a} \ln \tilde{\psi}_{o}(t), & \mu_{o} \geq \frac{\nu n \ln \left(\left(1+e^{t_{o} / \sigma}\right) / 2\right)}{r}\end{cases}
$$

where $\tilde{\psi}_{o}(t)=\sum_{k=0}^{\infty} \frac{(-a)^{k}}{k !}\left[1+\frac{k \ln \left(\left(1+e^{t / \sigma}\right) / 2\right)}{\left[n \ln \left(\left(1+e^{t_{o} / \sigma}\right) / 2\right)+\mu_{o}\right]}\right]^{-(r+\nu)}$

and $\tilde{\psi}_{M}(t)=\sum_{k=0}^{\infty} \frac{(-a)^{k}}{k !}\left[1+\frac{k r \ln \left(\left(1+e^{t / \sigma}\right) / 2\right)}{\left[n \ln \left(\left(1+e^{t_{o} / \sigma}\right) / 2\right)\right](r+\nu)}\right]^{-(r+\nu)}$.

\section{Robust Bayesian analysis under the sampling scheme of Bartholomew censoring with unknown shape and scale parameters}

Under the same set up as in Section 3, the predictive density corresponding to the prior $\pi(\sigma, \lambda)$ under the sampling scheme of Bartholomew comes out to be

$$
m(\underline{\mathbf{x}} \mid \pi)=(1-\epsilon) m\left(\underline{\mathbf{x}} \mid q_{o}\right)+\epsilon m(\underline{\mathbf{x}} \mid q), \quad 0<\lambda<\infty,
$$

where

$$
\begin{aligned}
m(\underline{\mathbf{x}} \mid q) & =\int_{\sigma=0}^{\infty} \int_{\lambda=0}^{\infty} P\left[N\left(t_{o}\right)=r \mid t_{o}\right] g(\lambda \mid \mu) P(\sigma) d \lambda d \sigma \\
& =\frac{\Gamma(r+\nu)}{\Gamma(\nu) r !} \frac{\mu^{\nu} \beta^{\alpha}}{\Gamma(\alpha)} \int_{0}^{\infty} \frac{\left[n \ln \left(\left(1+e^{t o / \sigma}\right) / 2\right)\right]^{r} \sigma^{-\alpha-1} \exp (-\beta / \sigma)}{\left[n \ln \left(\left(1+e^{t o / \sigma}\right) / 2\right)+\mu\right]^{r+\nu}} d \sigma .
\end{aligned}
$$

Again, to obtain, the ML-II Bayes estimate of $\lambda$ and $\sigma$ under the sampling scheme of Bartholomew, we need their marginal posterior under the base prior $q_{o}$ and the generic prior $q$. But, it is not possible to get explicit expressions for them. We, therefore, apply Gibbs sampler to get the samples from the marginal distributions of these parameters. For this the full conditionals of $\lambda$ given $\sigma$ is given by

$$
\hat{\pi}^{*}(\lambda \mid \sigma)=\hat{\eta} g_{o}^{*}(\lambda \mid \sigma)+(1-\hat{\eta}) \hat{g}^{*}(\lambda \mid \sigma), \quad 0<\lambda<\infty .
$$

Similarly, by combining the likelihood (2.1) with the prior (3.2), we get the conditional posterior density of $z^{*}(\sigma \mid \lambda)$ as

$$
z^{*}(\sigma \mid \lambda) \propto \sigma^{-\alpha-1} \exp \left[-\beta / \sigma-\lambda n \ln \left(\left(1+e^{t o / \sigma}\right) / 2\right)\right]\left[n \lambda \ln \left(\left(1+e^{t o / \sigma}\right) / 2\right)\right]^{r} .
$$

\section{Simulation study}

\subsection{When scale parameter is known}

In order to obtain the ML-II Bayes estimates of $\lambda$ under Type II censoring, we have generated 1000 samples using Monte Carlo simulation method, each of size 50 from GHLD with $\lambda=2$ and $\sigma=1.2$. For each sample we 
arranged the data in ascending order and considered a sample of first 25 and 30 observations, respectively. We have considered the values of the hyper-parameters of the base prior to be $\left(\mu_{o}=0.5, \nu=4\right)$ and $\left(\mu_{o}=1.5, \nu=4\right)$ and obtained the ML-II Bayes estimates of $\lambda$ under SELF and LINEX loss functions. We used the software $R$ (www.r-project.org) for the computations of functions in the various expressions.

For various values of $\epsilon$ the average ML-II posterior mean, posterior risk and average ML-II posterior variance under SELF and LINEX for $\lambda$ by considering Type II censoring for $r=25$ and 30 is presented in Table 1.

Table 1. Average ML-II posterior mean (posterior risk) under SELF and LINEX $(a=0.01)$ of $\lambda$.

\begin{tabular}{|c|c|c|c|c|c|c|}
\hline \multirow[b]{2}{*}{$r \downarrow$} & \multirow[b]{2}{*}{$\epsilon \downarrow$} & \multirow[b]{2}{*}{$\mu_{o} \rightarrow$} & \multicolumn{2}{|c|}{ SELF } & \multicolumn{2}{|c|}{ LINEX } \\
\hline & & & 0.5 & 1.5 & 0.5 & 1.5 \\
\hline \multirow{12}{*}{25} & 0.0 & & $2.3441(0.2211)$ & $2.1452(0.16)$ & $2.3431(1.1 \mathrm{e}-05)$ & $2.1446(8 \mathrm{e}-06)$ \\
\hline & & & 0.1971 & 0.1642 & & \\
\hline & 0.2 & & $2.1824(0.2297)$ & $2.1306(0.1669)$ & $2.1815(1.1 \mathrm{e}-05)$ & $2.13(8 \mathrm{e}-06)$ \\
\hline & & & 0.184 & 0.1632 & & \\
\hline & 0.4 & & $2.1449(0.2159)$ & $2.1177(0.1725)$ & $2.1441(1.1 \mathrm{e}-05)$ & $2.117(9 \mathrm{e}-06)$ \\
\hline & & & 0.1735 & 0.1617 & & \\
\hline & 0.6 & & $2.1272(0.207)$ & $2.1059(0.1771)$ & $2.1263(1 \mathrm{e}-05)$ & $2.1053(9 \mathrm{e}-06)$ \\
\hline & & & 0.1673 & 0.1601 & & \\
\hline & 0.8 & & $2.1166(0.2009)$ & $2.0952(0.181)$ & $2.1158(1 \mathrm{e}-05)$ & $2.0946(9 \mathrm{e}-06)$ \\
\hline & & & 0.1632 & 0.1582 & & \\
\hline & 1 & & $2.1096(0.1966)$ & $2.0853(0.1843)$ & $2.1088(1 \mathrm{e}-05)$ & $2.0847(9 \mathrm{e}-06)$ \\
\hline & & & 0.1602 & 0.1563 & & \\
\hline \multirow{12}{*}{30} & 0.0 & & $2.2639(0.1542)$ & $2.1267(0.1301)$ & $2.2631(8 \mathrm{e}-06)$ & $2.1262(7 \mathrm{e}-06)$ \\
\hline & & & 0.1553 & 0.1369 & & \\
\hline & 0.2 & & $2.1258(0.1584)$ & $2.1143(0.135)$ & $2.125(8 \mathrm{e}-06)$ & $2.1137(7 \mathrm{e}-06)$ \\
\hline & & & 0.1456 & 0.1361 & & \\
\hline & 0.4 & & $2.0957(0.1498)$ & $2.1031(0.1391)$ & $2.095(7 \mathrm{e}-06)$ & $2.1025(7 \mathrm{e}-06)$ \\
\hline & & & 0.1384 & 0.135 & & \\
\hline & 0.6 & & $2.0819(0.1446)$ & $2.0928(0.1425)$ & $2.0812(7 \mathrm{e}-06)$ & $2.0923(7 \mathrm{e}-06)$ \\
\hline & & & 0.1343 & 0.1338 & & \\
\hline & 0.8 & & $2.0738(0.1411)$ & $2.0834(0.1454)$ & $2.0732(7 \mathrm{e}-06)$ & $2.0829(7 \mathrm{e}-06)$ \\
\hline & & & 0.1317 & 0.1324 & & \\
\hline & 1 & & $2.0686(0.1387)$ & $2.0747(0.1479)$ & $2.0679(7 \mathrm{e}-06)$ & $2.0742(7 \mathrm{e}-06)$ \\
\hline & & & 0.1299 & 0.131 & & \\
\hline
\end{tabular}

Note: The second row represents the average ML-II posterior variance.

To investigate $R(t)$ and $h(t)$ under Type II censoring, we have generated 1000 samples using Monte Carlo simulation method, each of size 50 from GHLD with $\lambda=2$ and $\sigma=1$. For $r=25$, we obtained the ML-II Bayes estimates of $R(t)$ and $h(t)$ under SELF and LINEX loss functions, with the same values of the hyper-parameters as considered earlier.

In Table 2, $R(t)$, the average ML-II posterior mean and average variance for different values of $t, u_{o}$ and $\epsilon$ under SELF and Type II censoring are presented and in Table 3, $h(t)$, the average ML-II posterior mean and average ML-II posterior variance for different values of $t, u_{o}$ and $\epsilon$ under SELF and Type II censoring are presented. Also the average ML-II posterior mean for different values of $t$ under LINEX by considering Type II censoring for $R(t)$ and $h(t)$ are presented in Table 4 and Table 5. 
Table 2. Average ML-II posterior mean and average variance under SELF for $R(t)$.

\begin{tabular}{|c|c|c|c|c|c|c|c|c|}
\hline & & $\epsilon \rightarrow$ & 0 & 0.2 & 0.4 & 0.6 & 0.8 & 1 \\
\hline$t$ & $R(t)$ & $\mu_{o} \downarrow$ & & & & & & \\
\hline \multirow[t]{4}{*}{0.1} & 0.90258 & 0.5 & 0.8881 & 0.8955 & 0.8971 & 0.8979 & 0.8984 & 0.8987 \\
\hline & & & $4.00 \mathrm{E}-04$ & $4.00 \mathrm{E}-04$ & $3.00 \mathrm{E}-04$ & $3.00 \mathrm{E}-04$ & $3.00 \mathrm{E}-04$ & $3.00 \mathrm{E}-04$ \\
\hline & & 1.5 & 0.8967 & 0.8974 & 0.898 & 0.8986 & 0.8991 & 0.8995 \\
\hline & & & $3.00 \mathrm{E}-04$ & $3.00 \mathrm{E}-04$ & $3.00 \mathrm{E}-04$ & $3.00 \mathrm{E}-04$ & $3.00 \mathrm{E}-04$ & $3.00 \mathrm{E}-04$ \\
\hline \multirow[t]{4}{*}{0.5} & 0.57015 & 0.5 & 0.5271 & 0.5515 & 0.5568 & 0.5592 & 0.5607 & 0.5616 \\
\hline & & & 0.0038 & 0.0038 & 0.0037 & 0.0036 & 0.0035 & 0.0035 \\
\hline & & 1.5 & 0.5555 & 0.5579 & 0.5601 & 0.562 & 0.5638 & 0.5654 \\
\hline & & & 0.0036 & 0.0036 & 0.0036 & 0.0035 & 0.0035 & 0.0035 \\
\hline \multirow[t]{4}{*}{0.9} & 0.3342 & 0.5 & 0.2973 & 0.3245 & 0.3301 & 0.3326 & 0.3341 & 0.335 \\
\hline & & & 0.0042 & 0.0046 & 0.0045 & 0.0044 & 0.0044 & 0.0044 \\
\hline & & 1.5 & 0.3212 & 0.324 & 0.3265 & 0.3287 & 0.3307 & 0.3326 \\
\hline & & & 0.0044 & 0.0044 & 0.0044 & 0.0044 & 0.0044 & 0.0044 \\
\hline \multirow[t]{4}{*}{1.5} & 0.13312 & 0.5 & 0.1153 & 0.1351 & 0.1389 & 0.1405 & 0.1415 & 0.1421 \\
\hline & & & 0.0021 & 0.0026 & 0.0026 & 0.0026 & 0.0026 & 0.0025 \\
\hline & & 1.5 & 0.1334 & 0.1357 & 0.1377 & 0.1395 & 0.1411 & 0.1426 \\
\hline & & & 0.0024 & 0.0025 & 0.0025 & 0.0025 & 0.0026 & 0.0026 \\
\hline
\end{tabular}

Note: The first row represents the average ML-II posterior mean and second row represents the average variance.

Table 3. Average ML-II posterior mean and average variance under SELF for $h(t)$.

\begin{tabular}{|c|c|c|c|c|c|c|c|c|}
\hline & & $\epsilon \rightarrow$ & 0 & 0.2 & 0.4 & 0.6 & 0.8 & 1 \\
\hline$t$ & $h(t)$ & $\mu_{o} \downarrow$ & & & & & & \\
\hline \multirow[t]{4}{*}{0.1} & 1.04996 & 0.5 & 1.212 & 1.1266 & 1.1075 & 1.0986 & 1.0933 & 1.0899 \\
\hline & & & 0.0525 & 0.0489 & 0.046 & 0.0444 & 0.0434 & 0.0426 \\
\hline & & 1.5 & 1.1157 & 1.1078 & 1.1007 & 1.0943 & 1.0884 & 1.083 \\
\hline & & & 0.0443 & 0.044 & 0.0436 & 0.0431 & 0.0426 & 0.0421 \\
\hline \multirow[t]{4}{*}{0.5} & 1.24492 & 0.5 & 1.4516 & 1.3507 & 1.3276 & 1.3166 & 1.3102 & 1.3059 \\
\hline & & & 0.0753 & 0.0702 & 0.0662 & 0.0638 & 0.0623 & 0.0612 \\
\hline & & 1.5 & 1.3407 & 1.3317 & 1.3237 & 1.3164 & 1.3097 & 1.3036 \\
\hline & & & 0.0642 & 0.0638 & 0.0632 & 0.0626 & 0.0619 & 0.0611 \\
\hline \multirow[t]{4}{*}{0.9} & 1.4219 & 0.5 & 1.6348 & 1.5194 & 1.4937 & 1.4817 & 1.4746 & 1.47 \\
\hline & & & 0.0957 & 0.089 & 0.0839 & 0.081 & 0.079 & 0.0777 \\
\hline & & 1.5 & 1.5119 & 1.5013 & 1.4918 & 1.4832 & 1.4753 & 1.4681 \\
\hline & & & 0.0815 & 0.0809 & 0.0802 & 0.0793 & 0.0784 & 0.0774 \\
\hline \multirow[t]{4}{*}{1.5} & 1.63515 & 0.5 & 1.8889 & 1.7563 & 1.7266 & 1.7126 & 1.7043 & 1.6989 \\
\hline & & & 0.128 & 0.1192 & 0.1124 & 0.1084 & 0.1058 & 0.1039 \\
\hline & & 1.5 & 1.7574 & 1.7455 & 1.735 & 1.7254 & 1.7167 & 1.7087 \\
\hline & & & 0.1103 & 0.1096 & 0.1087 & 0.1076 & 0.1064 & 0.1051 \\
\hline
\end{tabular}

Note: The first row represents the average ML-II posterior mean and second row represents the average variance. 
Table 4. Average ML-II posterior mean under LINEX loss function for $R(t)$ for $a=0.01$.

\begin{tabular}{|c|c|c|c|c|c|c|c|c|}
\hline$t$ & \multicolumn{2}{|c|}{0.1} & \multicolumn{2}{|c|}{0.5} & \multicolumn{2}{|c|}{0.9} & \multicolumn{2}{|c|}{1.5} \\
\hline$R(t)$ & & & & 015 & & & & \\
\hline$\mu_{o} \rightarrow$ & 0.5 & 1.5 & 0.5 & 1.5 & 0.5 & 1.5 & 0.5 & 1.5 \\
\hline 0 & 0.8891 & 0.8971 & 0.5306 & 0.5514 & 0.2977 & 0.3233 & 0.1178 & 0.1307 \\
\hline 0.2 & 0.8965 & 0.8978 & 0.5551 & 0.5537 & 0.325 & 0.3261 & 0.1381 & 0.1329 \\
\hline 0.4 & 0.8982 & 0.8984 & 0.5603 & 0.5558 & 0.3305 & 0.3286 & 0.1419 & 0.1348 \\
\hline 0.6 & 0.8989 & 0.8989 & 0.5628 & 0.5577 & 0.3331 & 0.3309 & 0.1436 & 0.1366 \\
\hline 0.8 & 0.8994 & 0.8994 & 0.5642 & 0.5593 & 0.3345 & 0.3329 & 0.1445 & 0.1381 \\
\hline 1 & 0.8997 & 0.8999 & 0.5651 & 0.5609 & 0.3355 & 0.3348 & 0.1451 & 0.1396 \\
\hline
\end{tabular}

Table 5. Average ML-II posterior mean under LINEX loss function for $h(t)$ for $a=0.01$.

\begin{tabular}{|c|c|c|c|c|c|c|c|c|}
\hline $\mathrm{t}$ & \multicolumn{2}{|c|}{0.1} & \multicolumn{2}{|c|}{0.5} & \multicolumn{2}{|c|}{0.9} & \multicolumn{2}{|c|}{1.5} \\
\hline $\mathrm{h}(\mathrm{t})$ & \multicolumn{2}{|c|}{1.04996} & \multicolumn{2}{|c|}{1.24492} & \multicolumn{2}{|c|}{1.4219} & \multicolumn{2}{|c|}{1.63515} \\
\hline$\mu_{o} \rightarrow$ & 0.5 & 1.5 & 0.5 & 1.5 & 0.5 & 1.5 & 0.5 & 1.5 \\
\hline$\epsilon \downarrow$ & & & & & & & & \\
\hline 0 & 1.2028 & 1.1158 & 1.4475 & 1.3298 & 1.6413 & 1.5107 & 1.8793 & 1.738 \\
\hline 0.2 & 1.1174 & 1.1079 & 1.3468 & 1.3207 & 1.526 & 1.5 & 1.7467 & 1.7256 \\
\hline 0.4 & 1.0986 & 1.1009 & 1.3239 & 1.3126 & 1.5002 & 1.4905 & 1.7173 & 1.7147 \\
\hline 0.6 & 1.0898 & 1.0945 & 1.313 & 1.3052 & 1.4881 & 1.4819 & 1.7035 & 1.705 \\
\hline 0.8 & 1.0847 & 1.0887 & 1.3066 & 1.2985 & 1.481 & 1.474 & 1.6954 & 1.6961 \\
\hline 1 & 1.0813 & 1.0834 & 1.3024 & 1.2923 & 1.4763 & 1.4668 & 1.6901 & 1.6879 \\
\hline
\end{tabular}

Table 6. Average ML-II posterior mean (posterior risk) under SELF and LINEX $(a=0.01)$ of $\lambda$.

\begin{tabular}{|c|c|c|c|c|c|c|}
\hline \multirow{2}{*}{$t_{o} \downarrow$} & & \multirow{3}{*}{$\mu_{o} \rightarrow$} & \multicolumn{2}{|c|}{ SELF } & \multicolumn{2}{|c|}{ LINEX } \\
\hline & $\epsilon \downarrow$ & & 0.5 & 1.5 & 0.5 & 1.5 \\
\hline \multirow{12}{*}{0.3} & 0.0 & & $2.4076(0.2456)$ & $2.1356(0.1947)$ & $2.4059(1.2 \mathrm{e}-05)$ & $2.1345(1 \mathrm{e}-05)$ \\
\hline & & & 0.3372 & 0.2624 & & \\
\hline & 0.2 & & $2.1043(0.3303)$ & $2.1006(0.2218)$ & $2.1029(1.6 \mathrm{e}-05)$ & $2.0995(1.1 \mathrm{e}-05)$ \\
\hline & & & 0.2932 & 0.2591 & & \\
\hline & 0.4 & & $2.0429(0.3113)$ & $2.0725(0.2406)$ & $2.0416(1.6 \mathrm{e}-05)$ & $2.0714(1.2 \mathrm{e}-05)$ \\
\hline & & & 0.2641 & 0.2535 & & \\
\hline & 0.6 & & $2.0143(0.2986)$ & $2.0485(0.2549)$ & $2.013(1.5 \mathrm{e}-05)$ & $2.0475(1.3 \mathrm{e}-05)$ \\
\hline & & & 0.248 & 0.247 & & \\
\hline & 0.8 & & $1.9975(0.2901)$ & $2.0275(0.2663)$ & $1.9963(1.4 \mathrm{e}-05)$ & $2.0265(1.3 \mathrm{e}-05)$ \\
\hline & & & 0.2377 & 0.24 & & \\
\hline & 1 & & $1.9864(0.284)$ & $2.0086(0.2756)$ & $1.9853(1.4 \mathrm{e}-05)$ & $2.0076(1.4 \mathrm{e}-05)$ \\
\hline & & & 0.2305 & 0.2329 & & \\
\hline \multirow{12}{*}{0.5} & 0.0 & & $2.2658(0.1712)$ & $2.0847(0.1502)$ & $2.2649(9 \mathrm{e}-06)$ & $2.0839(8 \mathrm{e}-06)$ \\
\hline & & & 0.1889 & 0.1604 & & \\
\hline & 0.2 & & $2.0836(0.2054)$ & $2.0642(0.1626)$ & $2.0827(1 \mathrm{e}-05)$ & $2.0635(8 \mathrm{e}-06)$ \\
\hline & & & 0.1718 & 0.159 & & \\
\hline & 0.4 & & $2.0479(0.1974)$ & $2.0471(0.1717)$ & $2.0471(1 \mathrm{e}-05)$ & $2.0464(9 \mathrm{e}-06)$ \\
\hline & & & 0.1612 & 0.1569 & & \\
\hline & 0.6 & & $2.0317(0.1923)$ & $2.0323(0.1787)$ & $2.0309(1 \mathrm{e}-05)$ & $2.0316(9 \mathrm{e}-06)$ \\
\hline & & & 0.1556 & 0.1544 & & \\
\hline & 0.8 & & $2.0224(0.1889)$ & $2.0191(0.1843)$ & $2.0217(9 \mathrm{e}-06)$ & $2.0184(9 \mathrm{e}-06)$ \\
\hline & & & 0.1521 & 0.1517 & & \\
\hline & 1 & & $2.0164(0.1865)$ & $2.0072(0.1889)$ & $2.0156(9 \mathrm{e}-06)$ & $2.0065(9 \mathrm{e}-06)$ \\
\hline & & & 0.1498 & 0.1489 & & \\
\hline
\end{tabular}

Note: The second row represents the average ML-II posterior variance. 
In order to see the performance of estimators under the sampling scheme of Bartholomew, we generated 1000 samples each of size 50 from GHLD with $\lambda=2$ and $\sigma=1.2$. For each sample by fixing the time $t_{o}=0.3$ and $t_{o}=0.5$, we have calculated $r$ and then the estimates under SELF and LINEX loss functions. Since the results were following the same trend, therefore only the ML-II Bayes estimates of $\lambda$ are presented in Table 6 .

\subsection{When scale parameter is unknown}

\section{Algorithm}

This algorithm combines the Metropolis-Hastings with the Gibbs sampling scheme under the gamma proposal distribution.

1. Start with initial guess $\sigma^{(0)}$.

2. Set $i=1$.

3. Generate $\lambda^{(i)}$ from $\hat{\pi}^{*}\left(\lambda \mid \sigma^{(i-1)}\right)$ using (3.5).

4. Generated $\sigma^{(i)}$ from $z^{*}(\sigma \mid \lambda)$ using the Metropolis-Hasting algorithm with the proposal distribution $q(\sigma) \equiv$ inverted gamma $(\alpha, \beta)$.

4.1. Let $w=\sigma^{(i-1)}$

4.2. Generate prop from the proposal distribution $q$.

4.3. Let $p(w$, prop $)=\min \left\{1, \frac{z^{*}(\text { prop } \mid \lambda) q(w)}{z^{*}(w \mid \lambda) q(p r o p)}\right\}$.

4.4. Generate $u$ from $\operatorname{Uniform}(0,1)$. If $u \leq p(w, z)$ then accept the proposal and set $\sigma^{i}=z$; otherwise, set $\sigma^{i}=w$.

5. Set $i=i+1$;

6. Repeat Steps 3-5, $N$ times to obtain the posterior sample.

\section{Real data study}

Now we provide real data analysis to see how the model works in practice. We consider the failure log times to breakdown of an insulating fluid testing experiment (Nelson, 1982 and see Table 8). This data has been utilized by many authors, such as Balakrishnan and Kannan (2001), Balakrishnan et al. (2004), and Kim et al. (2011). Seo, Kim and Kang (2013), applying Kolmogorov test, showed that the data follow GHLD.

Table 7. Failure log times to breakdown of an insulating fluid testing experiment.

\begin{tabular}{cccccccc}
\hline 0.270027 & 1.02245 & 1.15057 & 1.42311 & 1.54116 & 1.57898 & 1.8718 & 1.9947 \\
2.08069 & 2.11263 & 2.48989 & 3.45789 & 3.48186 & 3.52371 & 3.60305 & 4.28895 \\
\hline
\end{tabular}

In order to apply Type II censoring scheme, we obtain first 12 lifetimes from the data and rest 4 observations are considered as censored. We consider the values of the hyper parameters of the base prior to be $\left(\mu_{o}=4, \nu=4\right)$ and $\left(\mu_{o}=8, \nu=4\right)$. As in this case both the shape and scale parameters are unknown, therefore we use the algorithm given in Section 6.2.

In order to obtain ML-II Bayes estimates under SELF and LINEX loss functions, we use Gibbs sampling technique. We observe from (2.10) and (2.11) that $g_{o}^{*}(\lambda \mid \sigma)$ and $g^{*}(\lambda \mid \sigma)$ both follow gamma distribution, therefore we obtain sample values form conditional posterior of $\lambda$ using rgamma() function available in $R$. However the conditional posterior for $\sigma$ does not follow any standard distribution. We therefore use MetropolisHastings algorithm to generate sample values for $\sigma$. We use inverted gamma distribution as proposal density. To obtain sample values from the proposal density, we use inverted gamma function available in MCMCpack in $R$. 
We run MCMC chain with a randomly chosen value of $\sigma$ and generate 50000 observations. To diminish the effect of the starting distribution, we discard the first 10000 observations and focus on the remaining 40000. The diagnostic plots for $\lambda$ and $\sigma$ for different values of $\epsilon$ and $\mu_{o}=4$ and $\mu_{o}=8$ are given in Figure 1 and 2, respectively.
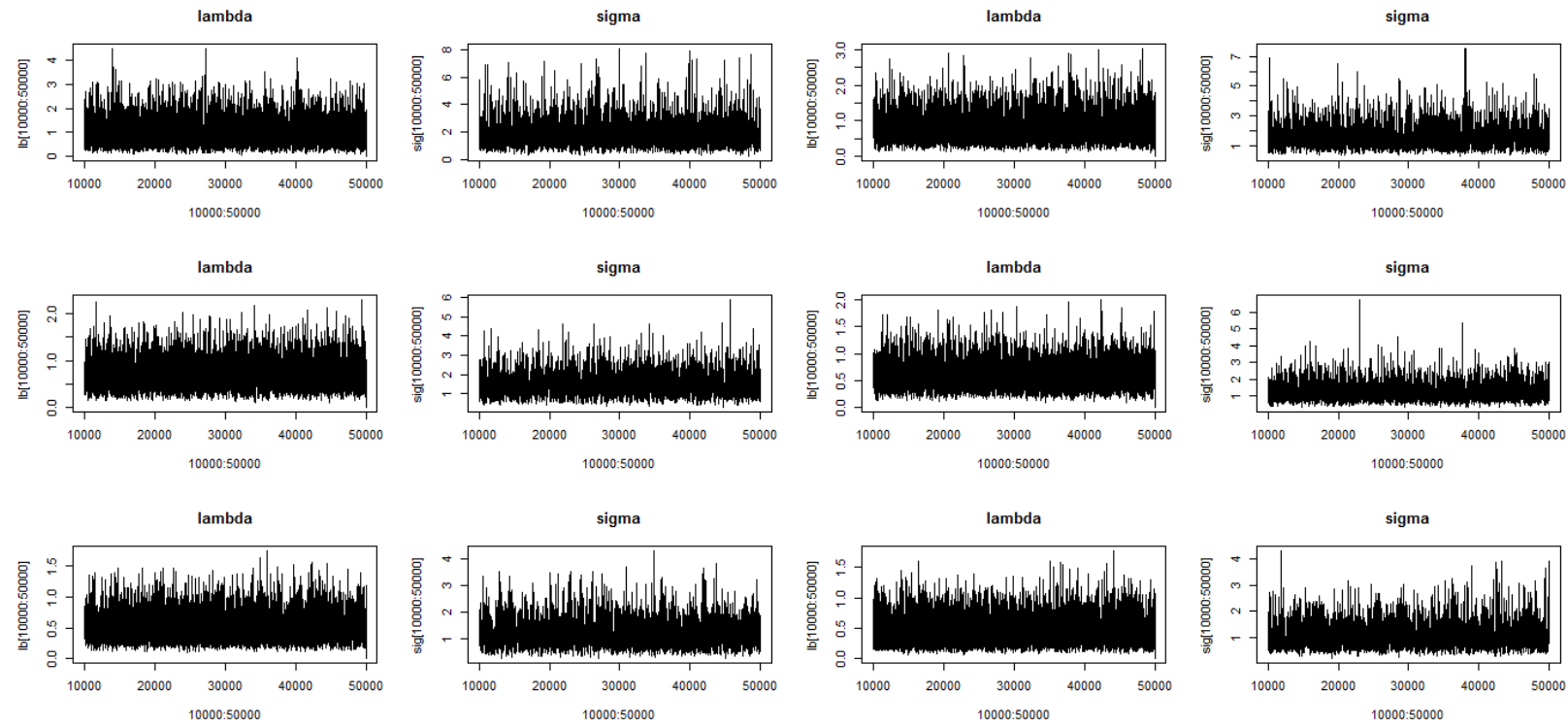

Figure 1. Diagnostic plots for $\lambda$ and $\sigma$ for $\mu_{o}=4$
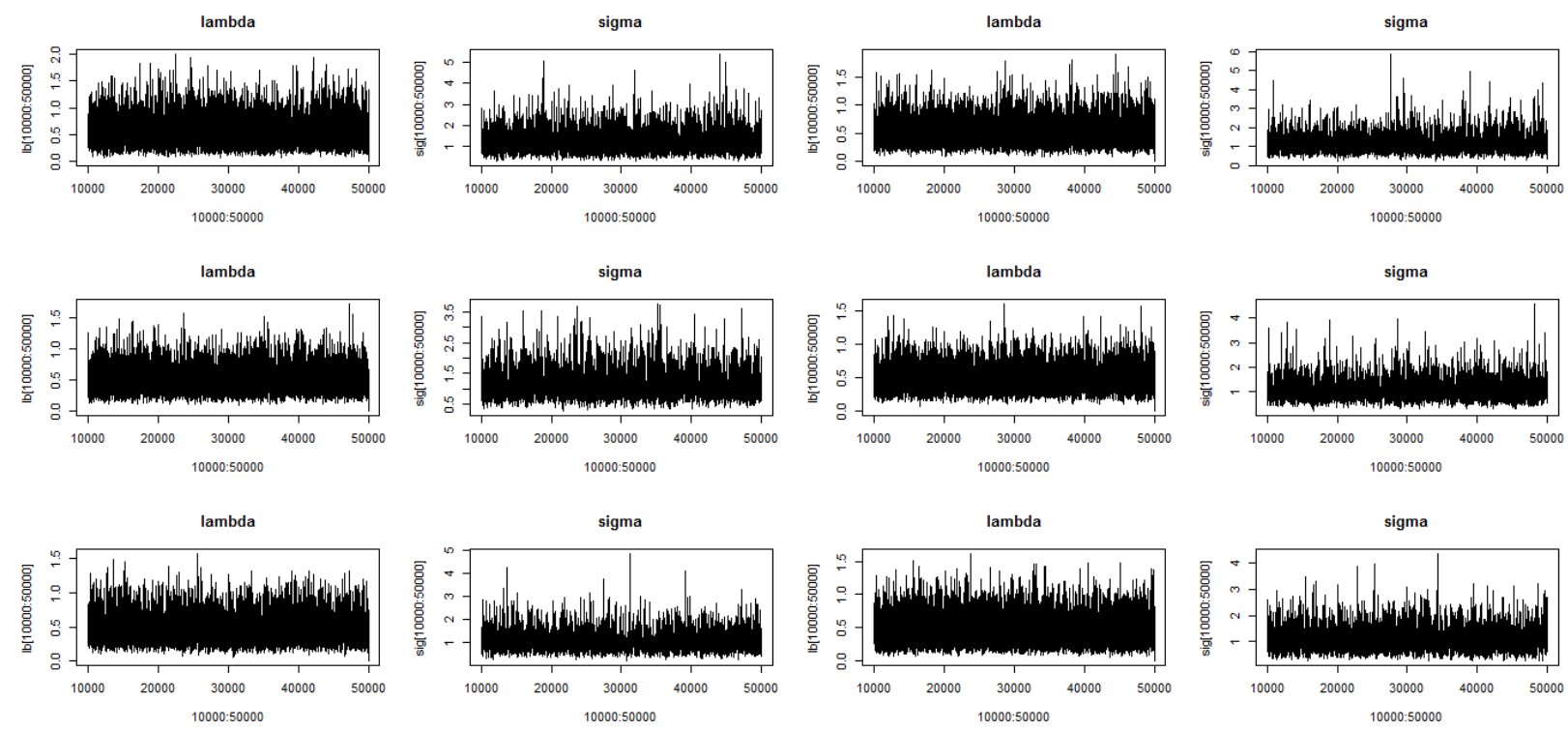

Figure 2. Diagnostic plots for $\lambda$ and $\sigma$ for $\mu_{o}=8$

The trace plot shows that the mean of the Markov chain is constant over the graph and is stabilized. The chain is able to traverse the support of the target distribution, and the mixing is good. The trace plot shows that the Markov chain appears to have reached a stationary distribution. 


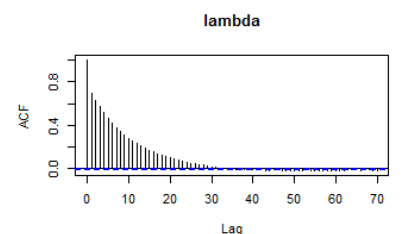

lambda

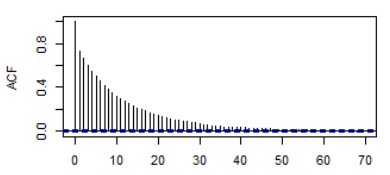

Lag

lambda

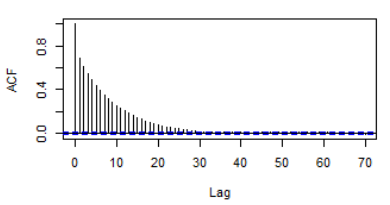

lambda

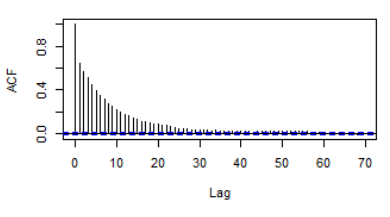

lambda

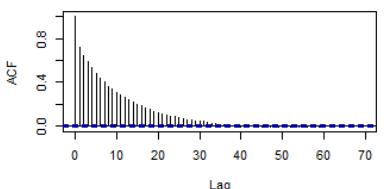

lambda

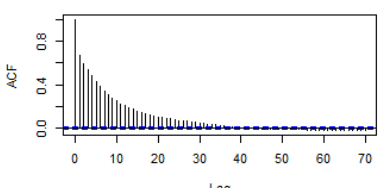

sigma

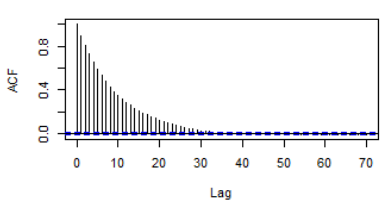

sigma

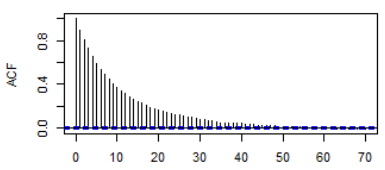

Lag

sigma

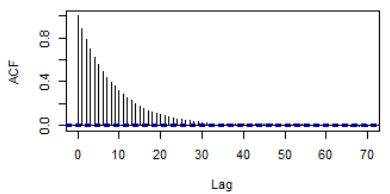

lambda

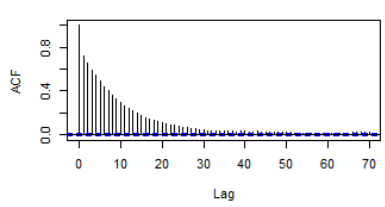

lambda

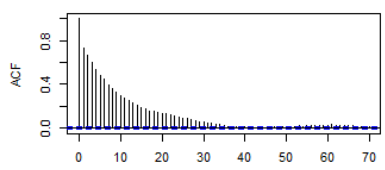

Lag

lambda

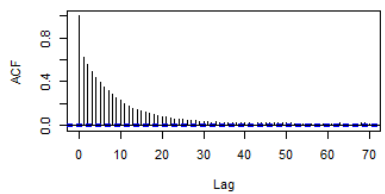

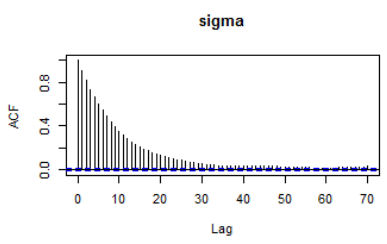

sigma

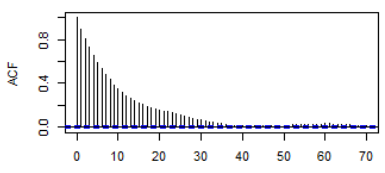

Lag

sigma

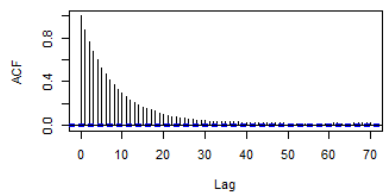

Figure 3. Auto-correlation plots for $\lambda$ and $\sigma$ for $\mu_{o}=4$
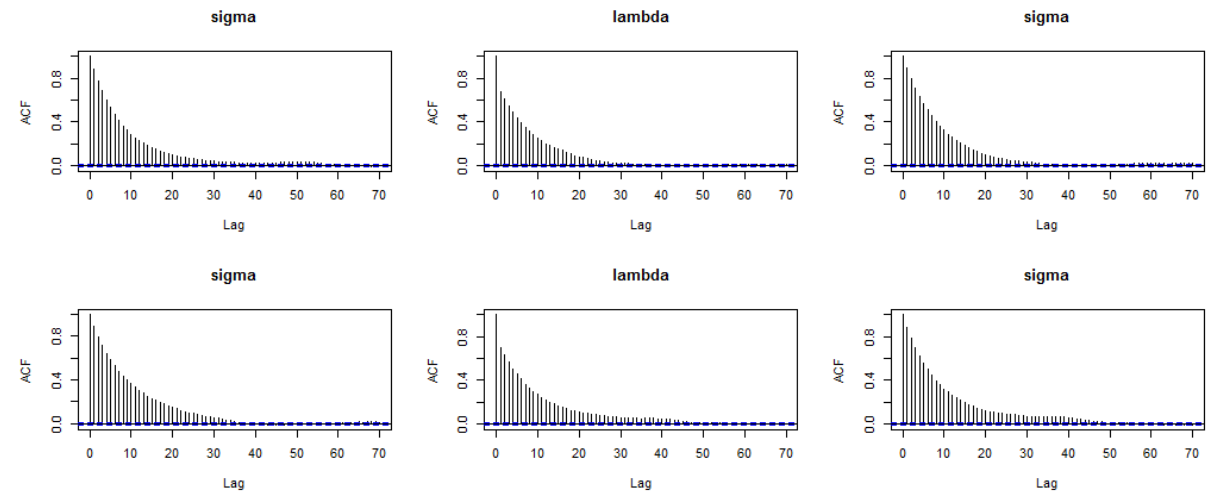

sigma
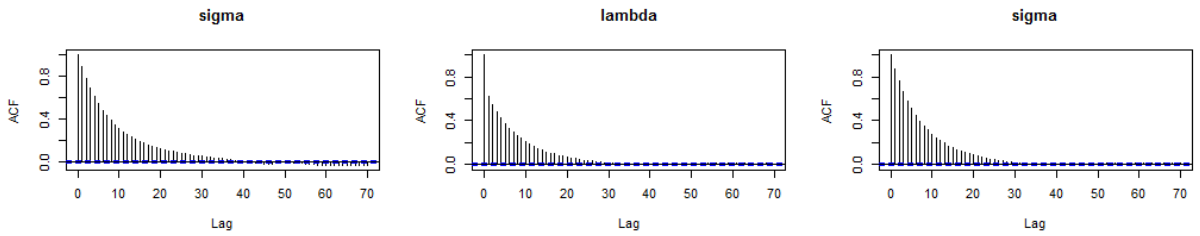

Figure 4. Auto-correlation plots for $\lambda$ and $\sigma$ for $\mu_{o}=8$

Using auto-correlation plots given in Figure 3 and in Figure 4 for $\mu_{o}=4$ and $\mu_{o}=8$ we conclude that we should pick every $50^{\text {th }}$ observation. Therefore, to provide relatively independent samples for improvement of prediction accuracy, we calculate the Bayesian MCMC estimates by the means of every sampled values after discarding the first 10000 values from 50000 .

In Figure 1, 2, 3 and 4, respectively, graph 1 and 2 are plotted for $\epsilon=0.0$, graph 3 and 4 are plotted for $\epsilon=0.2$, graph 5 and 6 are plotted for $\epsilon=0.4$, graph 7 and 8 are plotted for $\epsilon=0.6$, graph 9 and 10 are plotted for $\epsilon=0.8$, graph 11 and 12 are plotted for $\epsilon=1.0$. 
Table 8. ML-II posterior mean under SELF and LINEX for $\lambda$ and $\sigma$.

\begin{tabular}{|c|c|c|c|c|c|c|c|c|}
\hline \multirow[b]{3}{*}{$\mu_{o} \rightarrow$} & \multicolumn{4}{|c|}{ SELF } & \multicolumn{4}{|c|}{ LINEX } \\
\hline & \multicolumn{2}{|c|}{$\hat{\lambda}$} & \multicolumn{2}{|c|}{$\hat{\sigma}$} & \multicolumn{2}{|c|}{$\tilde{\lambda}$} & \multicolumn{2}{|c|}{$\tilde{\sigma}$} \\
\hline & 4 & 8 & 4 & 8 & 4 & 8 & 4 & 8 \\
\hline 0.0 & 0.91869 & 0.51275 & 1.79257 & 1.14045 & 0.91566 & 0.512 & 1.78243 & 1.13721 \\
\hline 0.2 & 0.76429 & 0.50146 & 1.57316 & 1.1182 & 0.76268 & 0.50087 & 1.56654 & 1.11537 \\
\hline 0.4 & 0.65742 & 0.48062 & 1.37591 & 1.08599 & 0.65632 & 0.48019 & 1.37081 & 1.08364 \\
\hline 0.6 & 0.57724 & 0.46549 & 1.25019 & 1.05408 & 0.57646 & 0.46507 & 1.24636 & 1.05183 \\
\hline 0.8 & 0.48223 & 0.44926 & 1.08714 & 1.01904 & 0.48166 & 0.44884 & 1.08427 & 1.01698 \\
\hline 1.0 & 0.43215 & 0.42207 & 1.00947 & 1.00275 & 0.43166 & 0.42156 & 1.00701 & 1.00008 \\
\hline
\end{tabular}

Table 9. Bayes estimates under SELF for $\epsilon=0.4$.

\begin{tabular}{|c|c|c|c|c|}
\hline \multirow{3}{*}{$\begin{array}{c}\mu_{o} \rightarrow \\
t \downarrow\end{array}$} & \multicolumn{2}{|c|}{$R(t)$} & \multicolumn{2}{|c|}{$Z(t)$} \\
\hline & 4 & 8 & 4 & 8 \\
\hline & & & & \\
\hline 1 & 0.75538 & 0.7589 & 0.32974 & 0.32732 \\
\hline 2 & 0.52889 & 0.5329 & 0.39207 & 0.38868 \\
\hline 4 & 0.23609 & 0.24134 & 0.44094 & 0.43129 \\
\hline 6 & 0.1027 & 0.10766 & 0.45371 & 0.44066 \\
\hline 8 & 0.04537 & 0.04902 & 0.4574 & 0.44297 \\
\hline 10 & 0.02074 & 0.0231 & 0.45858 & 0.44361 \\
\hline 15 & 0.00398 & 0.00461 & 0.45919 & 0.4439 \\
\hline 20 & 0.00167 & 0.00182 & 0.45926 & 0.44392 \\
\hline
\end{tabular}

\section{Discussion and conclusion}

From Table 1, we conclude that the posterior risk is decreasing as number of failures i.e., $r$ is increasing. Also from Table 1-3, it can be seen that ML-II posterior variance under SELF for $\lambda, R(t)$ and $h(t)$ is decreasing as epsilon is increasing. From Table 1-7, it can be seen that as epsilon is increasing ML-II estimators under the two considered base priors under SELF and LINEX loss functions by considering Type II censoring or the sampling scheme of Bartholomew comes near i.e., for, $\epsilon=0$ we get the Bayes estimate under the base prior. As the value of $\epsilon$ increases the estimator tends to include more part of the data dependent prior. All the estimates are showing that ML-II estimator are robust with respect to the change in base. From Table 6, it can be seen that under SELF and the sampling scheme of Bartholomew as termination time $t_{o}$ is increasing the posterior risk and ML-II posterior variance decreases. It is also seen from Table 6, that under LINEX loss function and the sampling scheme of Bartholomew as termination time $t_{o}$ is increasing the posterior risk decreases. From Table 1 and Table 6 we also conclude that under the sampling scheme of Bartholomew posterior risk and ML-II posterior variance are higher as compare to Type II censoring scheme. Thus we can say Type II censoring scheme provides better estimates. Also, as the posterior risk under LINEX loss function are lesser than the posterior risk under SELF, therefore LINEX loss function is better than SELF.

\section{Acknowledgment}

The authors are thankful to the editor and referees for their valuable suggestions and comments which led to considerable improvement in the original version 


\section{REFERENCES}

1. S.H. Arora, G.C. Bhimani, and M.N. Patel, Some results on maximum likelihood estimators of parameters of generalized half logistic distribution under Type-I progressive censoring with changing, International Journal of Contemporary Mathematical Sciences, vol. 5, pp. 685-698, 2010.

2. A. Asgharzadeh, R. Rezaie, and M. Abdi, Comparisons of methods of estimation for the half-logistic distribution, Selçuk J. Appl. Math., Special Issue, pp. 93-108, 2011.

3. R. Azimi, Bayesian estimation of generalized half logistic type-II doubly censored data, International Journal of Scientific World, vol. 1, pp. 57-60, 2013.

4. N. Balakrishnan, Order statistics from the half logistic distribution, Journal of Statistics and Computer Simulation, vol. 20, pp. 287-309, 1985

5. N. Balakrishnan, and A. Asgharzadeh, Inference for the scaled half-logistic distribution based on progressively type II censored samples, Comunication in Statistics-Theory and Methods, vol. 34, pp. 73-87, 2005.

6. N. Balakrsihnan, and A. Hossain, Inference for the Type-II generalized logistic distribution under progressive Type-II censoring, Journal of Statistical Computation and Simulation, vol. 77, pp. 1013-1031, 2007.

7. N. Balakrishnan, and N. Kannan, Point and interval estimation for the logistic distribution based on progressively Type-II censored samples, in Handbook of Statistics, N. Balakrishnan and C. R. Rao, Eds., vol. 20, pp. 431-456, 2001.

8. N. Balakrishnan, N. Kannan, C.T. Lin, and S.J.S. Wu, Inference for the extreme value distribution under progressively Type-II censoring, Journal of Statistical Computation and Simulation, vol. 74, pp. 25-45, 2004.

9. N. Balakrishnan, and N. Puthenpura, Best linear unbiased estimators of location and scale parameters of the half logistic distribution, Journal of Statistics and Computer Simulation, vol. 25, pp. 193-204, 1986.

10. D.J. Bartholomew, The sampling distribution of an estimate arising in life testing, Technometrics, vol. 5, pp. 361-374, 1963.

11. J.O. Berger, Bayesian robustness and Stein effect, J. Am. Stat. Assoc., vol. 77, pp. 358-368, 1982.

12. J.O. Berger, The robust Bayesian viewpoint (with discussion), In: Kadane, J. (ed.) Robustness in Bayesian Statistics, North Holland, 1983.

13. J.O. Berger, Statistical Decision Theory and Bayesian Analysis, New York: Springer-Verlag, 1985.

14. J.O. Berger, and J.M. Berliner, Robust Bayes and empirical Bayes analysis with $\epsilon$-contaminated priors, Technical Report No. 83-85, Department of Statistics, Purdue University, West Lafayette, 1983.

15. J.O. Berger, D.R. Insua, and F. Ruggeri, Bayesian robustness. In: Rios Insua, D., Ruggeri, F. (eds.), Robust Bayesian Analysis, Lecture Notes in Statistics, vol. 152, pp. 1-32. Springer, New York, 2000.

16. B. Betro, Numerical treatment of Bayesian robustness problems, Int. J. Approx. Reason., vol. 50, pp. 279-288, 2009.

17. G.E.P. Box, and G.C. Tiao, A further look at robustness in Bayes theorem, Biometrica, vol. 49, pp. 419-432, 1962.

18. G.E.P. Box, Sampling and Bayes inferences in scientific modeling and robustness (with discussion), J. Roy. Stat. Soc., vol. B41, pp. $113-147,1980$

19. A. Chaturvedi, Robust Bayesian analysis of the linear regression model, J. Stat. Plan. Infer., vol. 50, pp. 175-186, 1993.

20. A. Chaturvedi, Robust Bayesian analysis of the Exponential failure model, Pak. J. Stat. vol. 14, no. 24, pp. 115-126, 1998.

21. A. Chaturvedi, S.B. Kang, and A. Pathak, Estimation and testing procedures for the reliability functions of generalized half logistic distribution, Journal of The Korean Statistical Society, vol. 45, pp. 314-328, 2016.

22. A. Chaturvedi, M. Pati, and S.K. Tomer, Robust Bayesian analysis of Weibull failure model, METRON. DOI 10.1007/s40300-0130027-7, 2013.

23. A.P. Dempster, Examples relevant to the robustness of applied inferences, In: Gupta, S.S., Berger, J.O. (eds.) Stat. Decis. Theory Relat. Topics III. Academic Press, NY, 1976.

24. I.J. Good, Probability and the Weighting of Evidence, Griffin: London, 1950.

25. I.J. Good, The estimation of Probability, MIT Press, Cambridge, 1965.

26. I.J. Good, A Bayesian significance test for multinomial distributions, J. Roy. Stat. Soc., vol. B-29, pp. 399-443, 1967.

27. I.J. Good, Some history of the hierarchical Bayesian methodology, In: J.M. Bernardo, M.H. DeGroot, D.V. Lindley, A.F.M. Smith (eds.), Bayesian Statistics. University Press, Valencia, 1980.

28. H. Jeffreys, Theory of Probability, 3rd edn., Clarendon Press, Oxford, 1961.

29. Y. Kim, S.B. Kang, and J.I. Seo, Bayesian estimation in the generalized half logistic distribution under progressively Type II censoring, Journal of the Korean Data and Information Science Society, vol. 22, pp. 977-987, 2011.

30. D.V. Lindley, The robustness of interval estimates, Bull. Int. Stat. Inst., vol. 38, pp. 209-220, 1961.

31. W.B. Nelson, Applied Life Data Analysis, John Willey and Sons, New York, 1982.

32. J.I. Seo, and S.B. Kang, Entropy estimation of generalized half-logistic distribution (GHLD) bassed on Type-II censored samples, Entropy, vol. 16, pp. 443-454, 2014.

33. J.I. Seo, Y. Kim, and S.B. Kang, Estimation on the generalized half logistic distribution under Type-II hybrid censoring, Communications for Statistical Applications and Methods, vol. 20, pp. 63-75, 2013.

34. J.I. Seo, H.J. Lee, and S.B. Kang, Estimation for generalized half logistic distribution based on records, Journal of the Korean Data and Information Science Society, vol. 23, pp. 1249-1257, 2012. 